\title{
Research Paper \\ Structural Pattern of Death Attitude Based on Attachment Styles in Adolescents With Cancer: Mediator Role of Repression and Anxiety Sensitivity
}

\author{
*Mojtaba Ahmadi Farsani ${ }^{1} \odot$, Rasool Heshmati², Touraj Hashemi Nosrat Abad ${ }^{3}$
}

1. MA. in Child and Adolescence Clinical Psychology, Department of Psychology, Faculty of Educational Science and Psychology, University of Tabriz, Tabriz, Iran 2. PhD. in Health Psychology, Faculty Member, Department of Psychology, Faculty of Educational Science and Psychology, University of Tabriz, Tabriz, Iran.

3. PhD. in Educatonal Psychology, Professor, Department of Psychology, Faculty of Educational Science and Psychology, University of Tabriz, Tabriz, Iran

\begin{tabular}{l|l}
$\begin{array}{c}\text { Use your device to scan } \\
\text { and read the artice online }\end{array}$ \\
Styles in Adolescents With Cancer: Mediator Role of Repression and Anxiety Sensitivity (Persian)]. Iranian Journal of Psychiatry \\
and Clinical Psychology. 2019; 25(3):308-327. http://dx.doi.org/10.32598/ijpcp.25.3.308 \\
d 6 http://dx.doi.org/10.32598/ijpcp.25.3.308
\end{tabular}

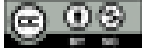

Received: 15 Oct 2018 Accepted: 14 Apr 2019 Available Online: 01 Oct 2019

Key words: Cancer, Attachment styles, Repression

\section{ABSTRACT}

Objectives Cancer is the fourth leading cause of death in adolescents. Psychological factors play an important role in the development and sustainability, and treatment outcomes of cancer. One of these factors is the attitude towards death. One of the variables that play a fundamental role in the attitude towards death is the quality of the parent-child relationship, which forms the attachment style basis. Therefore, in this research, the structural pattern of death attitude based on attachment styles in adolescents with cancer was explored. Accordingly, we investigated the mediating role of emotional repression and anxiety sensitivity.

Methods In total, 150 adolescents with cancer ( 85 girls \& 65 boys) in the Isfahan Province, Iran, referring to Health centers and Ala Cancer Prevention and Control Center (MACSA). They were selected by convenience sampling method. Then, they were evaluated by the following measures: Hazan and Shaver Attachment Style in Adults Inventory, Wong Death Attitude Inventory, Weinberger Emotional Repression Inventory, Anxiety Sensitivity (AS) Inventory. The obtained data were analyzed by Structural Equation Modeling (SEM).

Results The collected result indicated that attachment quality has a direct and significant relationship with the attitude toward death in adolescents with cancer. Additionally, attachment quality through the mediating role of emotional suppression and anxiety sensitivity had significant relationships with the attitude toward death in this population.

Conclusion Attachment styles, emotional suppression, and anxiety sensitivity are the important determinants of attitude toward death in adolescents with cancer. Moreover, the assessed model could explain the attitude toward death in these individuals.

\section{Extended Abstract}

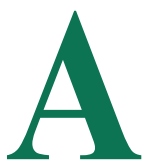

\section{Introduction}

ccording to the American Cancer Society (ACS), there is no precise definition of how childhood cancers separate from cancer in adolescents or the exact time that a person with cancer is considered an adult; however for statistical purposes, cancer in adolescents is often referred to people aged 15-19 years. About 5,000 adolescents (15-19 years old) in the United States are annually diagnosed with cancer, and about 600 adolescents die from cancer yearly.

Cancer causes about $5 \%$ of deaths and is the fourth cause of death in this age group after accidents, suicide, and mur-

* Corresponding Author:

Mojtaba Ahmadi Farsani, MA.

Address: Department of Psychology, Faculty of Education \& Psychology, University of Tabriz, Tabriz, Iran.

Tel: +98 (914) 7843368

E-mail: mojtaba.farsani94@gmail.com 
der. Psychological factors play an important role in the development of cancer and sustainability and outcomes of the disease treatment. Patients' expectations for illness and positive or negative attitudes toward illness and death affect their mortality rates, compliance rates, medical prescriptions, hospitalization, and recovery rates.

Attitude towards death refers to the total cognitive, emotional, and behavioral responses of a person to death. These attitudes can be positive or negative; negative attitudes about death include fear of death or avoidance of death. However, positive attitudes toward death include coping with death and accepting it. Coping with death consists of a set of cognitive, behavioral, and emotional responses to death before their own and others' death.

It is specified that coping with death is negatively correlated with the indicators of fear of death. Therefore, not only the identification of the attitude of people with cancer to death is important, but it is also necessary to identify the factors affecting death and its attitudes, such as attachment styles, emotional suppression, and anxiety sensitivity. One of the variables that play a fundamental role in the attitude towards death is the quality of the parent-child relationship, which forms the basis of the attachment style.

Attachment is the deep emotional bond that we establish with special people in our lives so that when we interact with them, we feel excited and relaxed, and when it comes to stress, feel relaxed about having them next to us. Attachment is vital for children; because as the child explores the surrounding world and faces unpredictable things, it gives them a sense of security and tranquility.

The attachment has different styles and is generally divided into two categories of secure and insecure. Evidence suggests that adolescents and adults enjoy secure-sharing patterns with greater satisfaction and long-standing relationships with others; while, insecure attachment is linked with emotional distances from others, loneliness, and hostility. Attachment contributes to the formation of the personality and traits; therefore, it is closely related to the way a person with cancer copes with his illness and follows the medical orders regimen. Insecure attachment imposes more stress on the person. When an adolescent with insecure attachment faces conditions like cancer, he is very disturbed and may have a negative attitude toward the future and death.

The lack of experiencing emotions, shedding feelings within the self, the lack of expressing emotions, and ultimately emotional repression can negatively affect the attitude to death. Emotional repression is a defense mechanism that reduces emotional pain; however, fails to help to resolve the real issue.

The repression of painful emotions by reinterpreting events makes us less aware of these unplanned things that we are incapable of changing and thinking about. Previous research has explored personality types and related illnesses in the field of health psychology. They concluded that cancer-prone persons tend to be depressed, provide desperate feedbacks, and fail to express emotions, especially the negative ones. This pattern forms a particular personality type, called the $\mathrm{C}$ personality. The findings suggested a close relationship between emotion suppression, which is one of the most essential characteristics of the $\mathrm{C}$ personality type and cancer.

Anxiety and its related problems, like high anxiety sensitivity, can also affect the death attitude. Anxiety sensitivity is a cognitive structure that was first considered by Reis and McLenn (1985). Anxiety sensitivity is practically a fear of anxiety and emotion associated with arousal. Individuals with high anxiety levels perceive stressful situations with extreme feelings and thoughts. Moreover, in extreme cases, they are prepared to respond to intense anxiety responses to stressful situations, where people with high anxiety sensitivity often react negatively to anxiety symptoms. However, people with low anxiety sensitivity, although they may perceive symptoms as unpleasant, do not consider them as a threat. Cancer is a threat, and many people are anxious to respond to it. In addition, the anxiety sensitivity level in coping with cancer and the attitude to death plays an important role in this area.

The role of attachment styles on death attitude is unclear in adolescence. Additionally, the role of emotional repression and anxiety sensitivity in the relationship between attachment styles and death attitude is undetermined. Thus, this research investigated the impact of these variables on the death attitude of adolescents with cancer in the form of a hypothesized model (Figure 1).

\section{Methods}

This was a fundamental and descriptive-correlational study. The study population consisted of adolescents with cancer in Isfahan City, Iran. They were selected by convenience sampling method $(\mathrm{N}=150)$. To collect data, Hazan and Shaver Attachment Styles in Adults Inventory, Wittenberg's Emotional Suppression Questionnaire Floyd Anxiety Sensitivity (AS) Inventory, and Wong Death Attitude Inventory were used. The obtained data were analyzed by Structural Equation Modeling (SEM). 


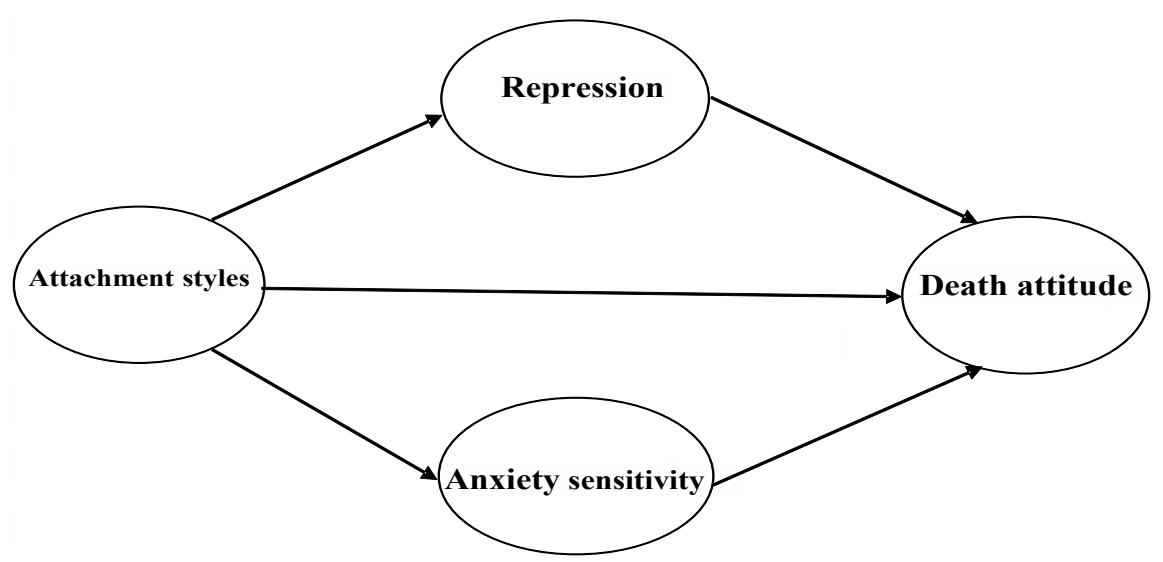

Figure 1. Hypothesized model

PSYCHIATRY AND CUNICAL PSYCHOLOGY

\section{Results}

The achieved results indicated that attachment styles have a significant direct relationship with the attitude toward death in adolescents with cancer $(\beta=0.40)$. Furthermore, attachment styles, through mediating role of emotional suppression and anxiety sensitivity, have significant relationships with the attitude toward death in adolescents with cancer.

\section{Discussion}

The study findings suggested that attachment, as an exogenous variable, affects the death attitude. In other words, the attentive care provided by the parents to the children and the appropriate response to their needs forms a secure attachment leading to schemas in them. Moreover, the world of creation, being and non-being, is a safe place. Such schema could influence the children's lives in the coming years (from adolescence to adulthood) and develops a sense of deep psychological security in person. Thus, even when faced with unpleasant and life-threatening events, including cancer and death, they fail to receive safety from the person. Because they consider death as a temporary separation from his security base (parent or caretaker). In addition, as a result of this security, the person is not afraid of death and has a positive view of it and accepts it. In people with insecure attachment, the opposite case happens.

The findings revealed that attachment styles, through the mediating role of emotional repression, have significant relationships with the death attitude in adolescents with cancer. In other words, an insecure attachment style (i. e. perceiving the world an unsafe) affects the subsequent growth of the individual; because the child is afraid of separating from the security base (parent or caregiver). As a result, there is no chance to discover a new environment and develop more social relationships and skills.

Therefore, he or she is deprived of learning adaptive strategies of emotion regulation, like emotional expression in life events. Instead, they use conflicting strategies, like emotional repression. Because of this repression and erroneous emotional regulation, one avoids coping with issues, like death, which creates a negative emotion or represses these issues and excitement in them. Accordingly, repressing the emotions and not having to cope with such issues gives them the chance to think about the subject to normalize it. As a result, they either avoid these issues or are afraid of thinking about them. In people with secure attachment styles, the opposite case happens.

The findings suggested that attachment styles, through the mediating role of anxiety sensitivity have significant relationships with the death attitude in adolescents with cancer.

Insecure attachment leads to mental insecurity in the child. Following this issue, the person interacts with the environment and does not separate from the security database. Therefore, he is deprived of learning various skills or lacks these skills. The lack of skills in tedium over time impacts child growth. These effects include cognitive problems (cognitive errors, attention bias, the formation of negative thoughts and irrational beliefs, etc. ), emotional shaking and emotional instability, or even impaired autonomic nervous system (sympathetic and parasympathetic) as a result of hormonal changes. Accordingly, the anxiety system of the person associated with these problems is disturbed, and the person generates anxiety problems, such as anxiety disorders, high anxiety sensitivity, and so on.

Along with the high sensitivity of anxiety, stressful events, like cancer and its unknown outcome (therapeutic or death) 
occur with many biopsychological symptoms. Because of the high anxiety sensitivity, he takes the symptoms as threats and magnifies them. As a result, to prevent the occurrence of these symptoms, the person attempts to deny the truth of cancer and the resulting death. As a result, he does not face cancer or death in general, and normalization does not occur. The final result is the tragedy of having to die and having a negative attitude toward it (fear and avoidance of death).

In conclusion, attachment styles, emotional suppression, and anxiety sensitivity are essential determinants of attitude toward death in adolescents with cancer. Moreover, the assessed model can explain the attitude toward death in these individuals.

\section{Ethical Considerations}

\section{Compliance with ethical guidelines}

An informed consent was obtained from the participants after explaining the research objectives and method; They were also free to leave the study at any time and were assured of the confidentiality of their information.

\section{Funding}

This research received no specific grant from funding agencies in the public, commercial, or not-for-profit sectors.

\section{Authors contributions}

Conceptualization, methodology, initial draft preparation by all authors; investigation, supervision and project administration by Mojtaba Ahmadi Farsani and Rasool Heshmati; resources, editing and review by Mojtaba Ahmadi Farsani.

\section{Conflicts of interest}

The authors declared no conflicts of interest.

\section{Acknowledgements}

the authors would like to thank all the participants as well as the management and personnel of the ALA Cancer Prevention and Control Center and Omid hospital of Isfahan for their cooperation in this research. 
This Page Intentionally Left Blank 


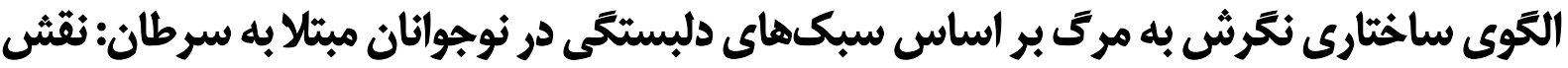

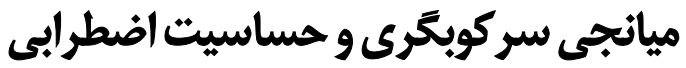

\author{
"مجتبى احمدى فارسانى' ، رسول حشمتى"، تورج هاشمى نصرتآباد"

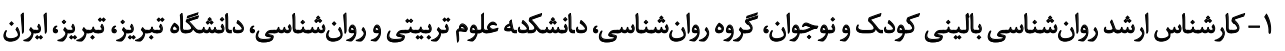

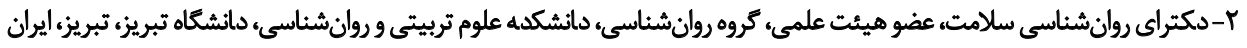

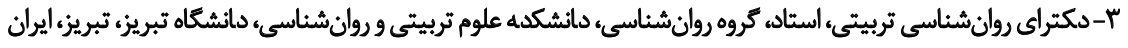

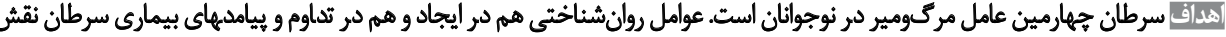

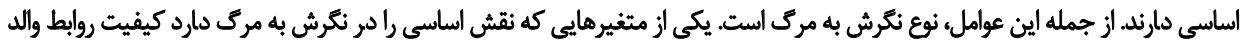

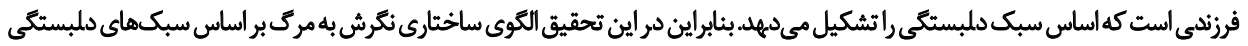

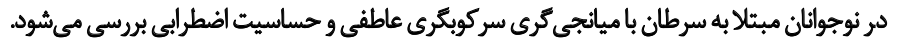

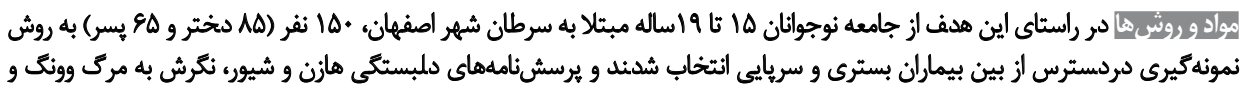

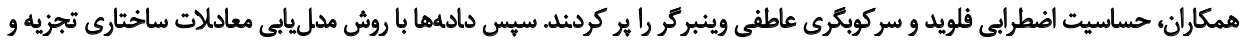
تحليلشدند.

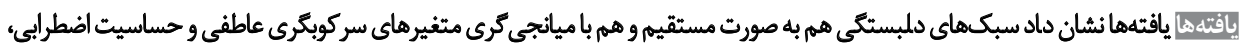

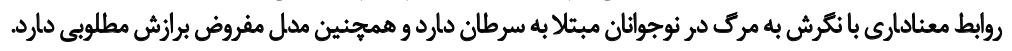

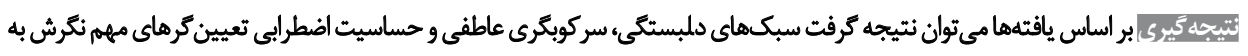

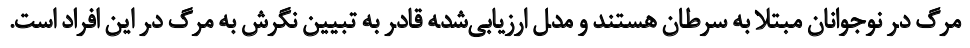

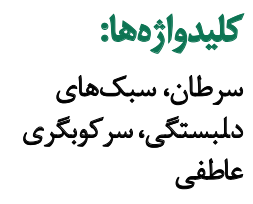

كليدوازوها:

سرطان، سبكهاي عاطفى

تداوم و هم در ييامدهاي بيمارى نقش اساسى دارند [ب] إنتظار

dale

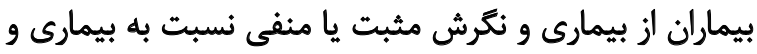

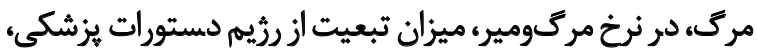
طول دوره بسترى و بهبودى اين بيماران تأثير دارد.

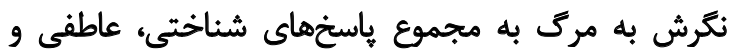

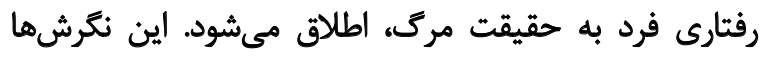

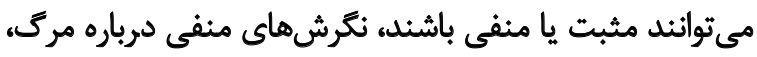

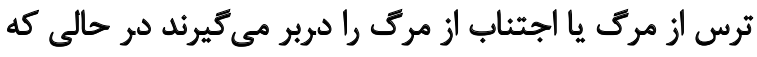

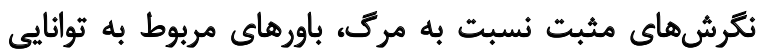

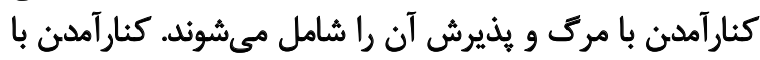

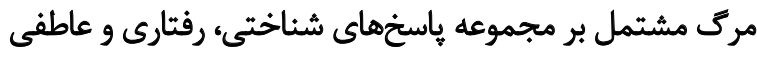
به مرك يشيروى خود و ديكران است.

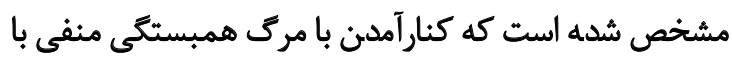

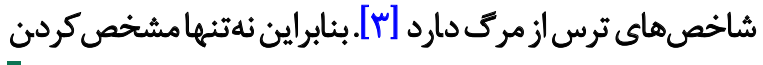

طبق تزارشهاى انجمن سرطان آمريكا' هيج تعريف دقيقى

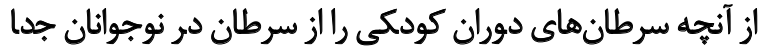

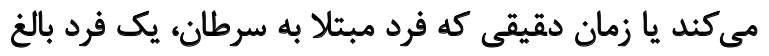

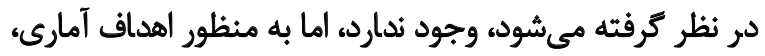

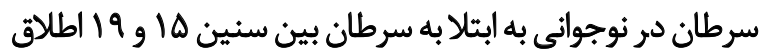

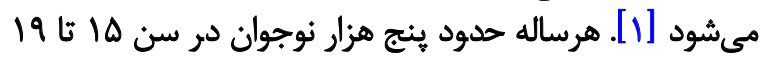

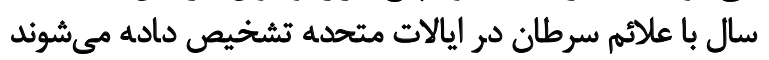

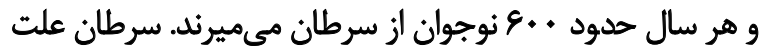

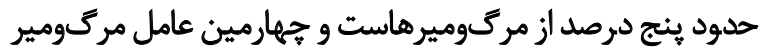

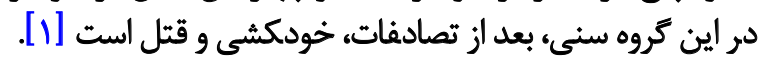
عوامل روانشناختى هم در ايجاد بيمارى سرطان و هم در

1. American Cancer Society (ACS)

* نويسنده مسئول:

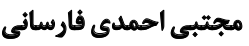

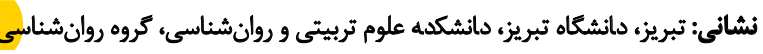

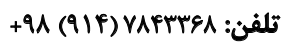
يست الكترونيكي: Mojtaba. farsani94@gmail. com 
روانشناسى سلامت، مشخص كردهاند شخصيت مستعد سرطان

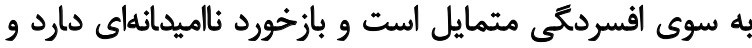

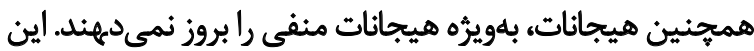

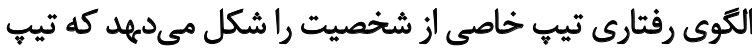

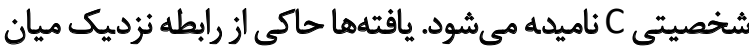

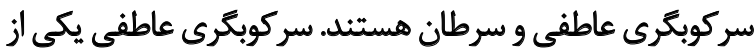

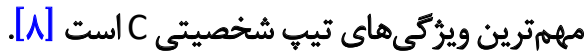
اضطراب و مشكلات مربوط به آن مانند بالابودن حساسيت

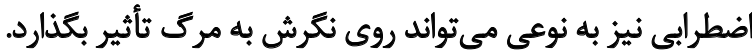

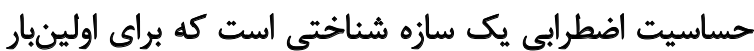

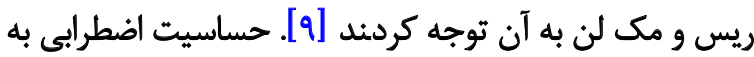
لحاظ عملى ترس از اضطراب و احساسات مرتبط با برانكيختئى

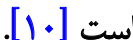

افراد با سطوح حساسيت اضطرابى بالا موقعيتهاى تنشزار را

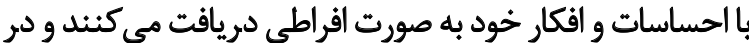

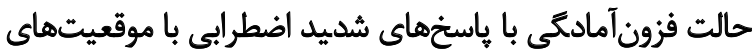

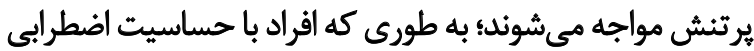

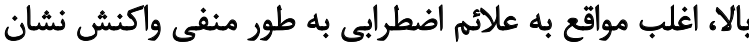

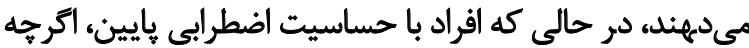

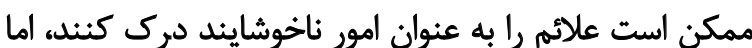

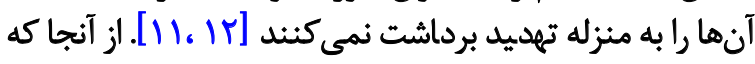

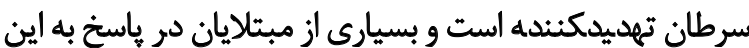

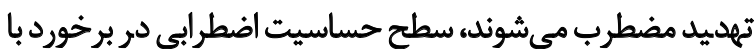
سرطان و نوع نكرش فرد مبتلا به مرك نقش مهمى دئ دارد. تحقيقات زيادى وجود دارند كه رابطه بين سرطان و دلبستئى

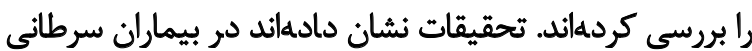

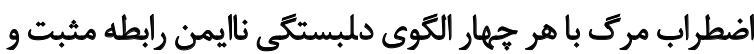

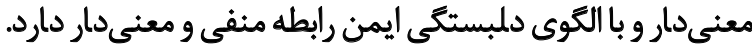

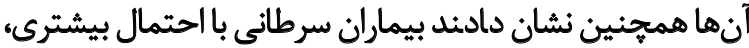

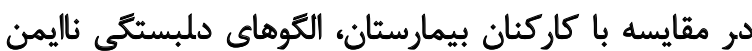

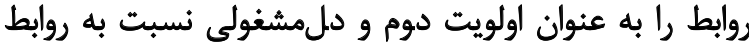

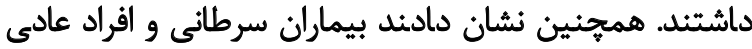

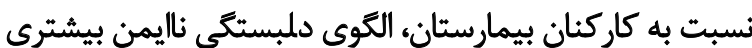

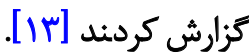

همجنين تحقيقات زيادى رابطه بين سرطان و انتظار مرى و ورئ

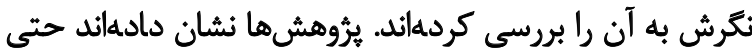

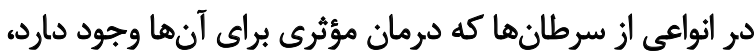

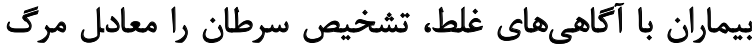

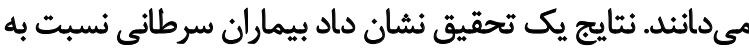

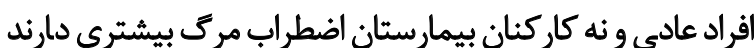

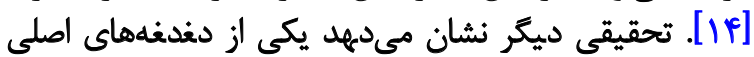

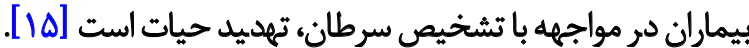

نكرش افراد مبتلا به سرطان به مرك اهميت دارد، بلكه شُناسايي

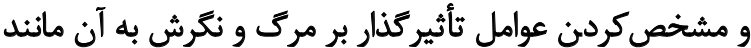

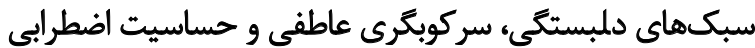
نيز ضرورى است. يكى از متغيرهايى كه نقش اساسى دئى در نترش

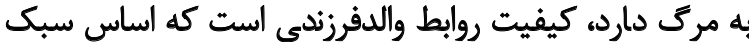
دلبستخى را تشكيل مي ميتهد.

دلمبستى عبارت است از ييوند عاطفى عميقى كه با افراد

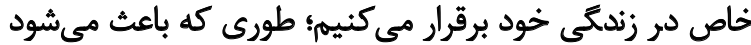

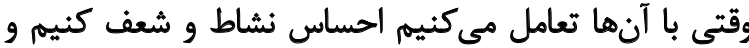

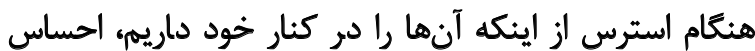

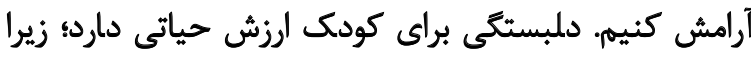

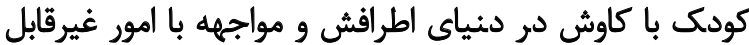

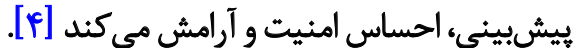

دلمبستى سبكهاي مختلفى دارد و به طور كلى بلى به دو نو

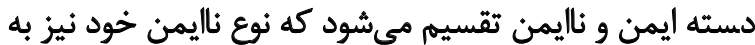

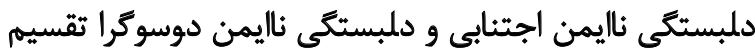

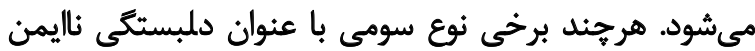

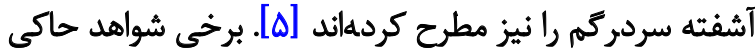

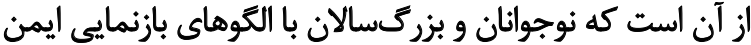

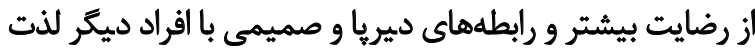

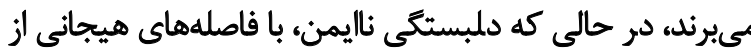

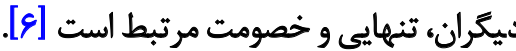

از آنجا كه دلبستكى در شكل تيرى شخصيت بُعدى فرد و

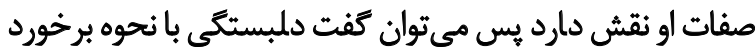

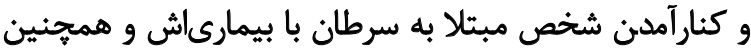

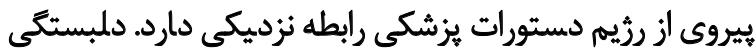

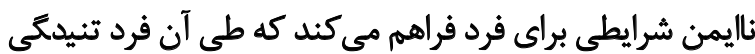

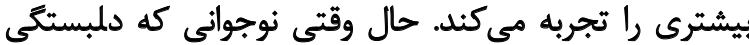

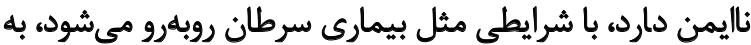

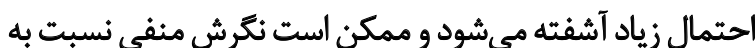

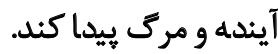

به نظر مىرسد تجربهنكردن عواطف، ريختن احساسات

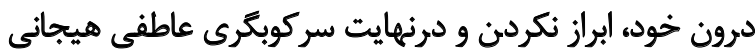

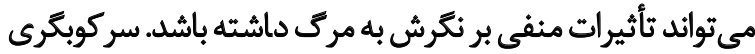

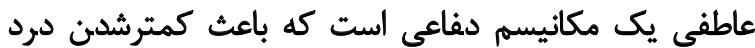

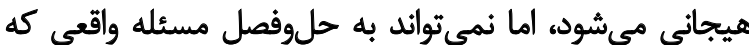

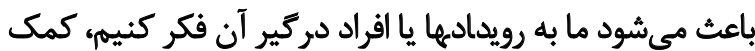

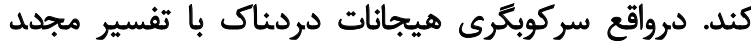

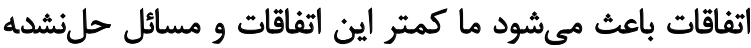

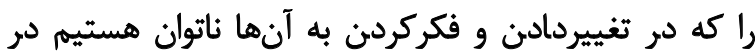

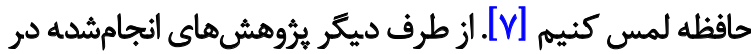

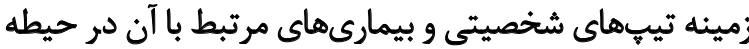




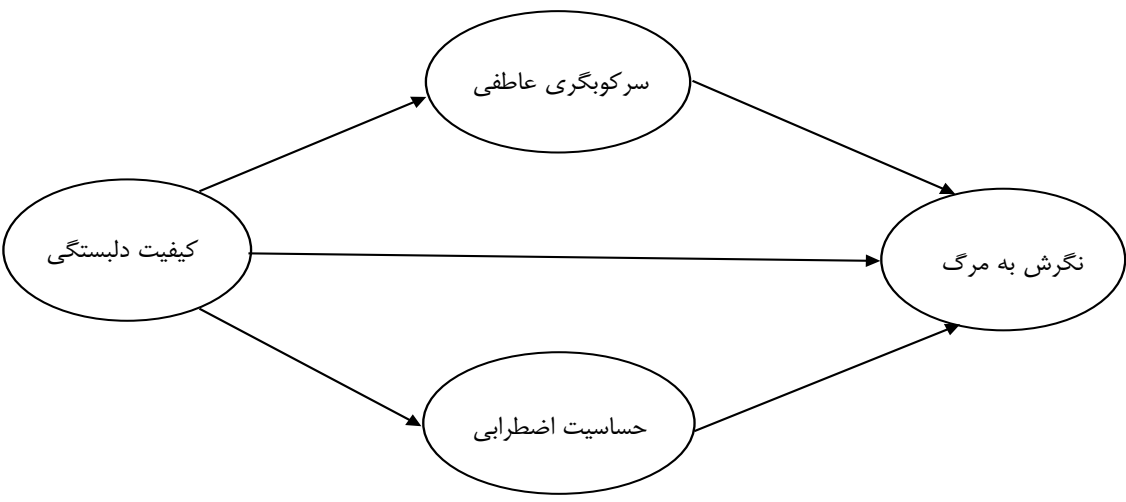

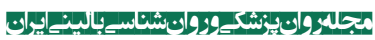

تصوير ا. مدل مفروض نكرش به مرك در نوجوانان مبتلا به سرطان

و اضطراب ممكن است اثرات مستقيمى بر دفاعهاى ايمنى بدن

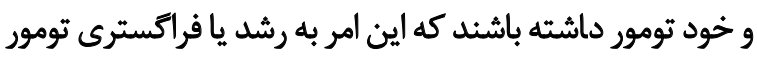

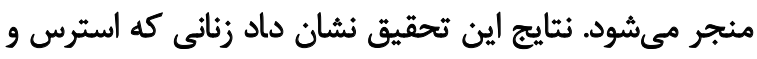

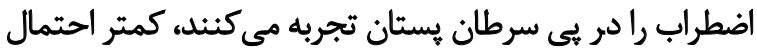

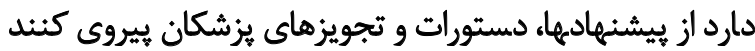

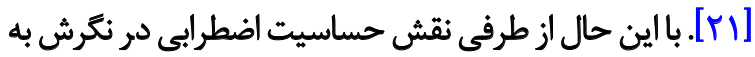

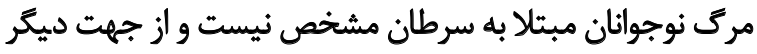

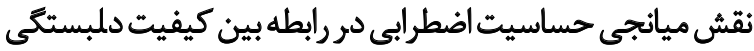

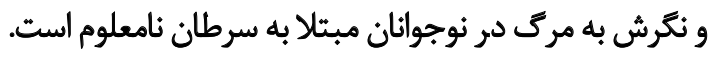

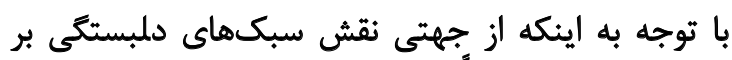

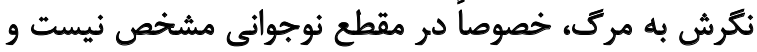

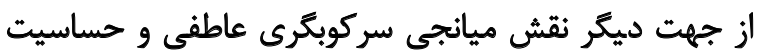

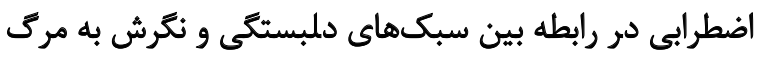

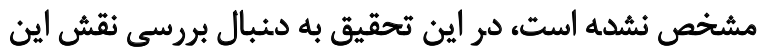

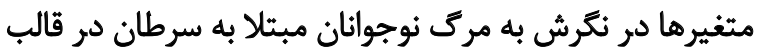

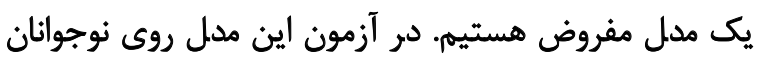

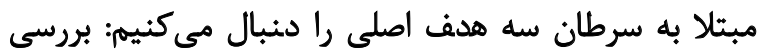

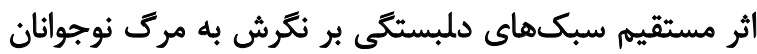

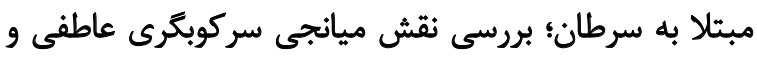

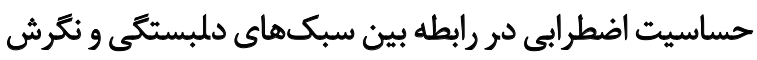

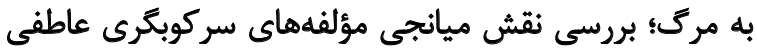

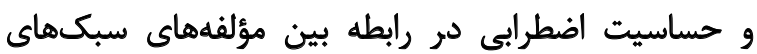

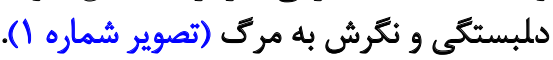

وروش

يُروهش حاضر از نوع توصيفىهمبستكى و مقطعى است.

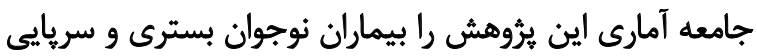

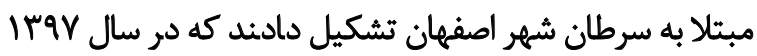

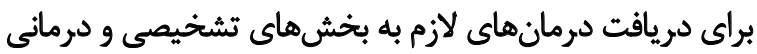

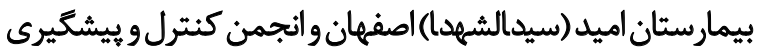

همجنين تحقيقات نُشان ميديهند بيماران سرطانى افسرديكى

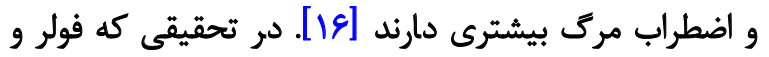

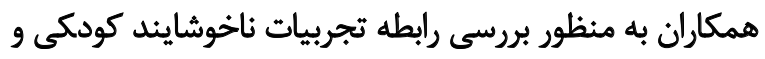

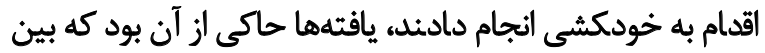
اقدام به خودكشى و تجربيات منفى و ناخوشايند كودكى إنى رابطه

معنى دارى وجود دارد [IV]

تحقيقات بسيارى انجامشده كه رابطه بين سركوبكرى عاطفي

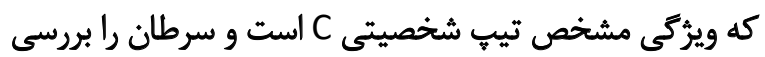

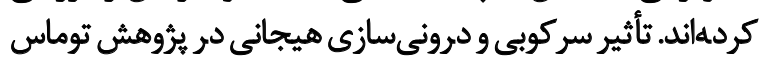

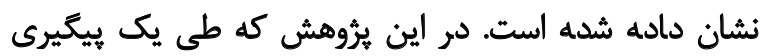

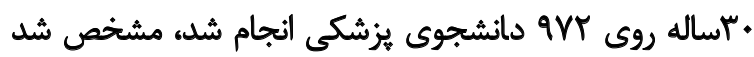

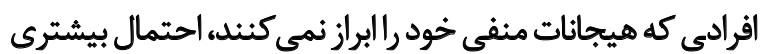

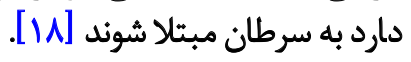

همجنين در تحقيق كايلو و همكاران كه به منظور بررسى نقش رئش

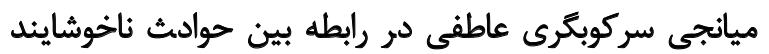

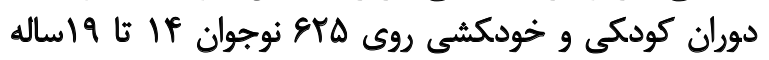

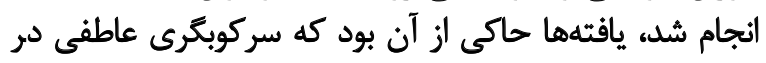

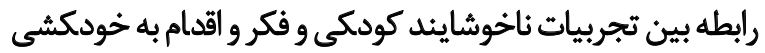
در نوجوانى نقش ميانجى دارد [19]

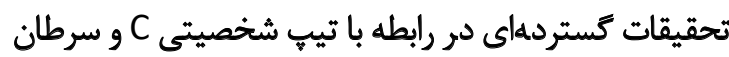

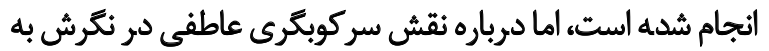

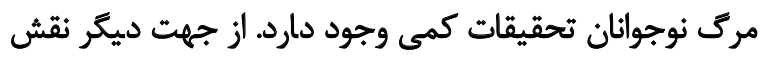

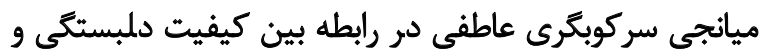

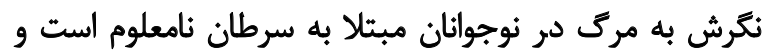
مشخص نشده است. تحقيقات بسيارى نيز روى رابطه سرطان و اضطراب و و مواب

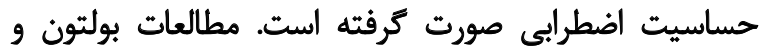

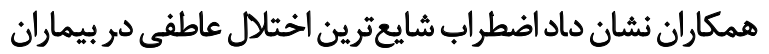

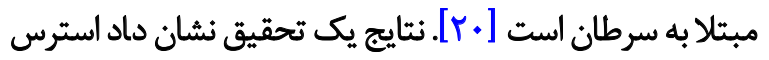


يرسشنامه به ترتيب ه ه و إست. ضريب اسيب آلفاي كرونباخ

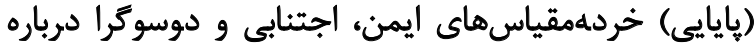

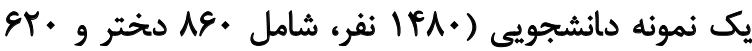

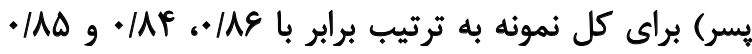

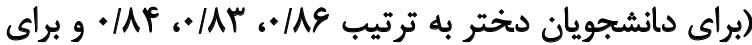

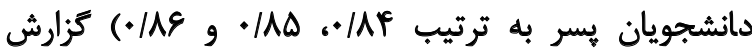

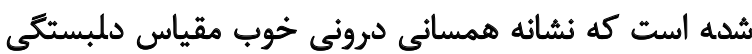

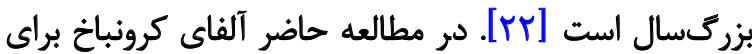

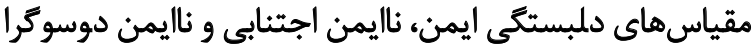

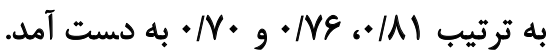

\section{يرسش نامه حساسيت اضطر ابى"}

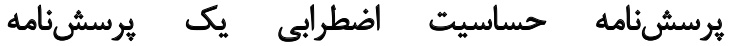

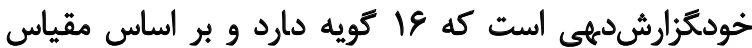

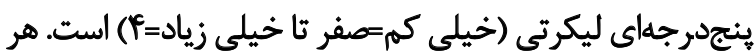

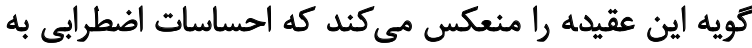

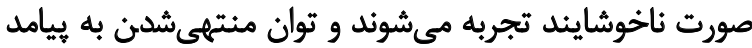

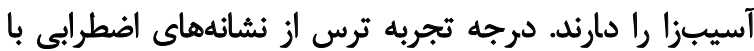

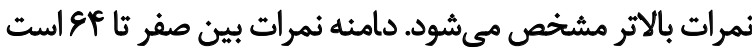

[rr]

ساختار اين يرسشنامه از سه عامل ترس از نكرانىهاي بدنى

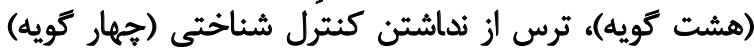

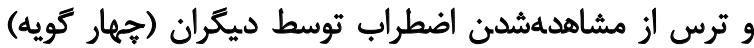

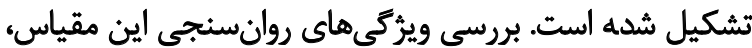

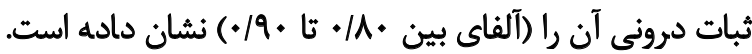

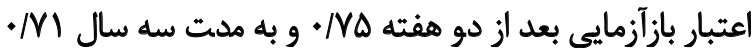

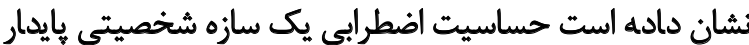

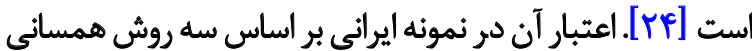

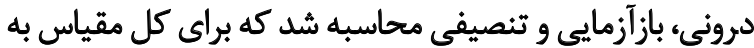

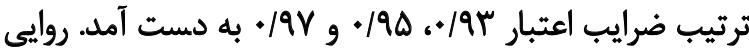

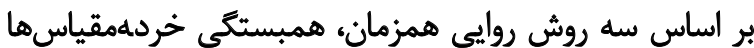

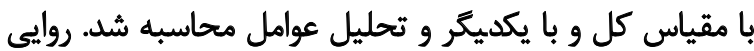

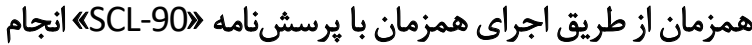

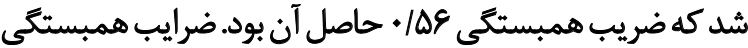

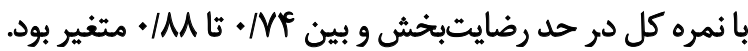

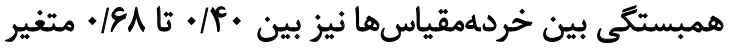

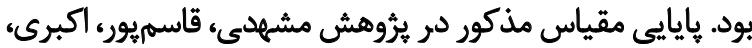

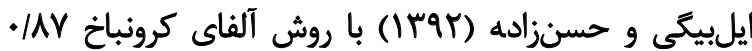

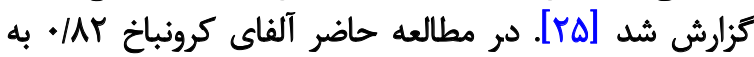
دست آمد.

$$
\text { از سرطان آلا مراجعه كرده بودند. }
$$

نمونه لازم براي تحقيق حاضر، • اه نفر در نظر ترفته شد كه

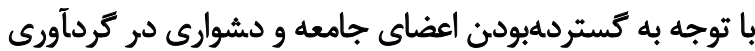

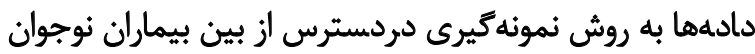

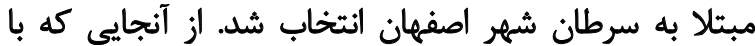

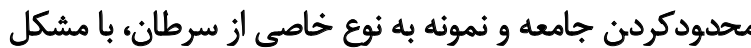

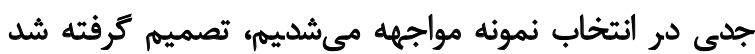

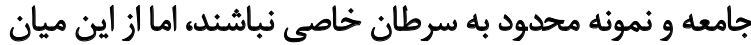

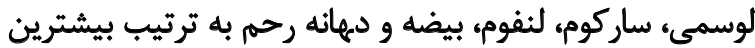
فراوانى را در نمونه يُروهش داشتيند.

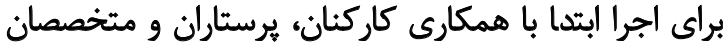

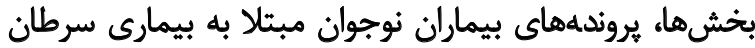

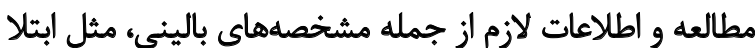

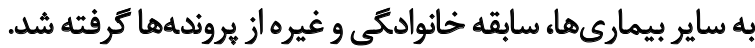

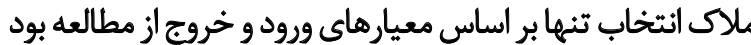

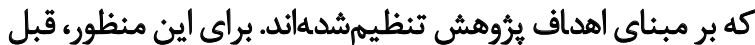

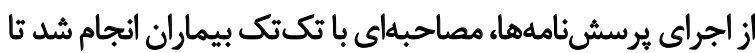

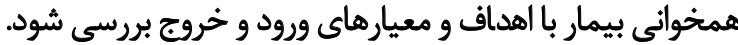
معيارهاى ورود عبارت بودند از: تشخيص اصلى بيمارى

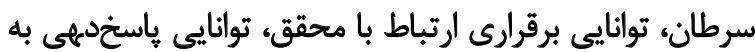

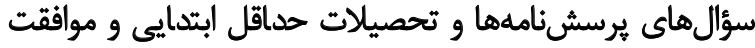

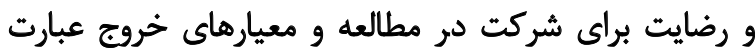

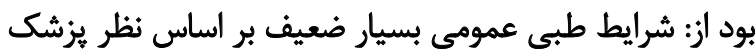

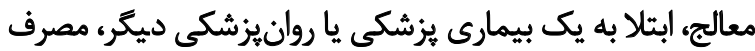

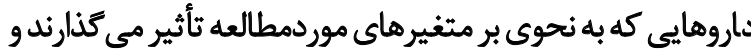

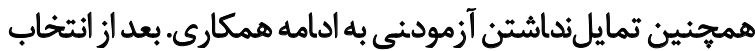

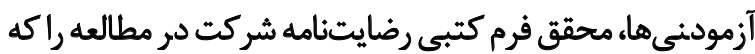

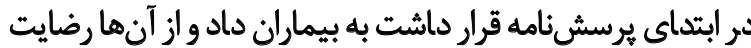

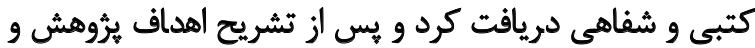

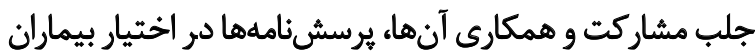

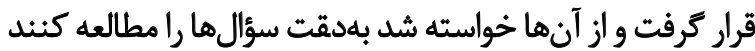

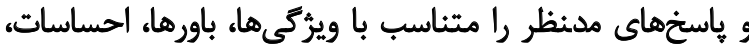

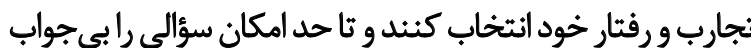

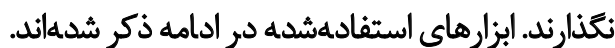

مقياس دلبستَّى بزروّىسالان

مقياس دلمبستى بزركسالان كه با استفاده از مواد

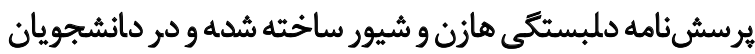

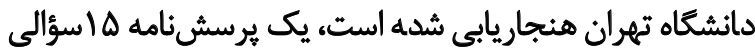

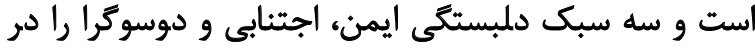

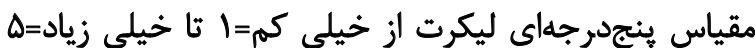

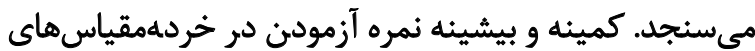


معادلات ساختارى" آزمايش شد. تحليل دادهها با استفاده از

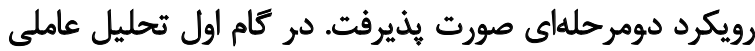

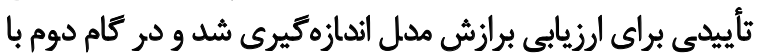

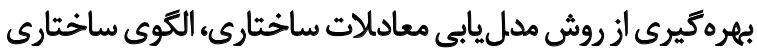

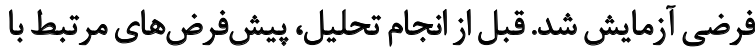

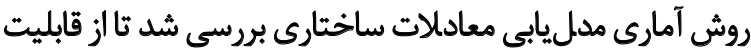

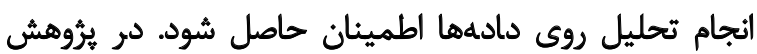

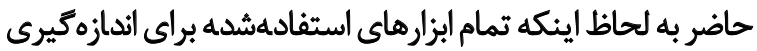

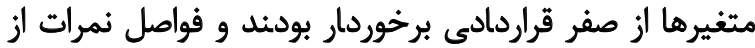

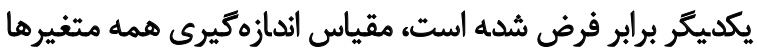

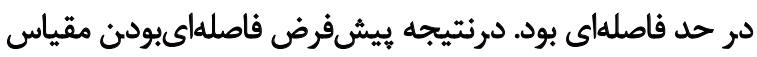

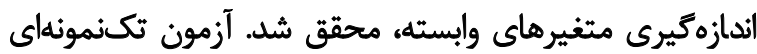

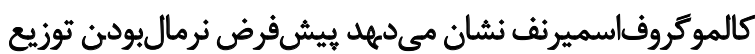

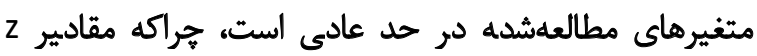

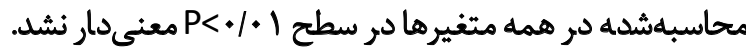
شاخص تحمله و شاخص تورم واريانس" نشان دادند

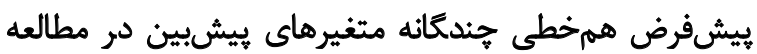

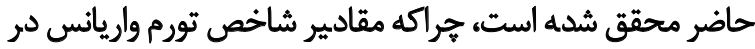

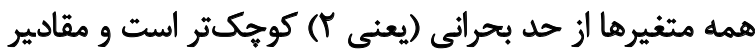

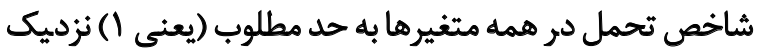

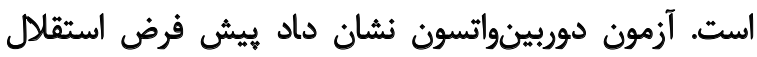

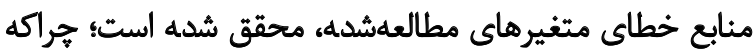

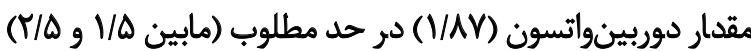

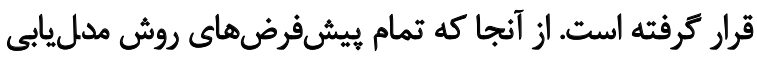

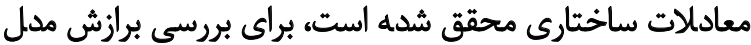

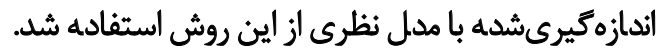

يأثتهها

ميانكين سنى براى كل كروه IV/TA بود. نمونه شامل هA

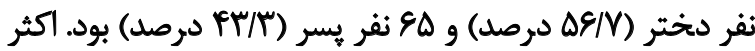

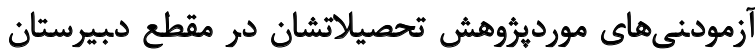

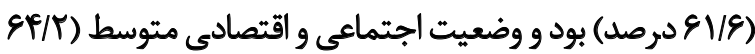

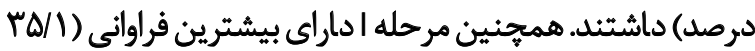

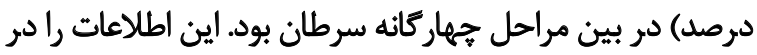

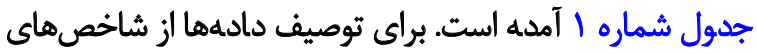

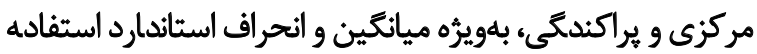

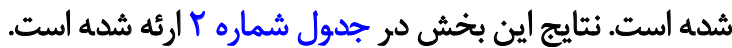

بر اساس يافتههاى مدليابى معادلات ساختارى مى معتوان

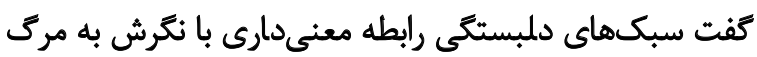

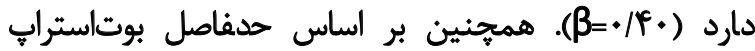

4. Structural Equation Modeling (SEM)

5. Tolerance

6. Variance Inflation Factor (VIF)

\section{يرسشنامه سركوبكرى عاطفى وينبرتر"}

اين يرسشنامه از يرسشنامه سازكارى وينبركر ترفته

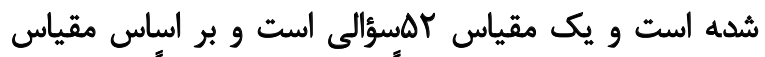

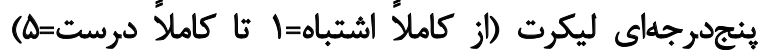

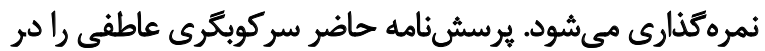

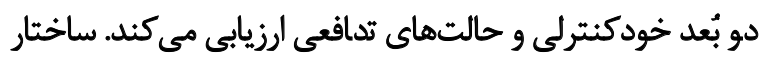

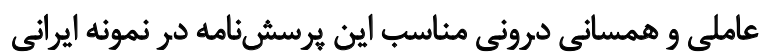

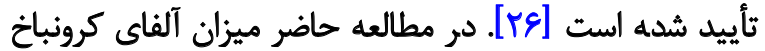

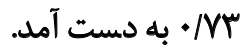
فرم تجديدنظرشده نتّرش نسبت به مرتى

اين آزمون را وونكَ، بيكر و كيسر در سال $199 f$ ساختهاند.

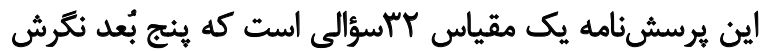

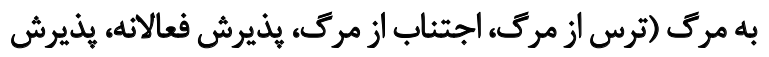

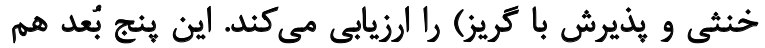

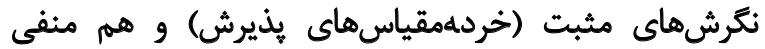

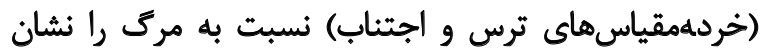

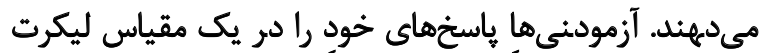

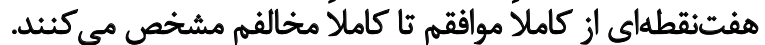

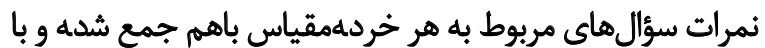

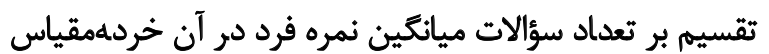

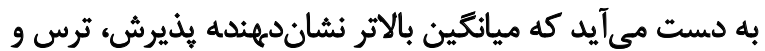

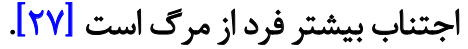

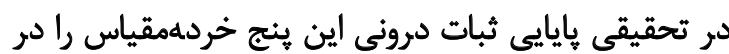

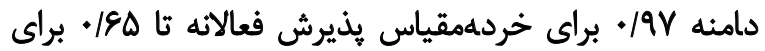

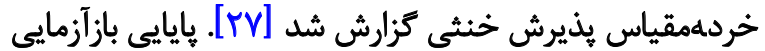

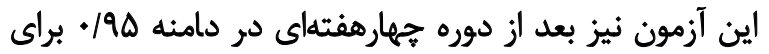

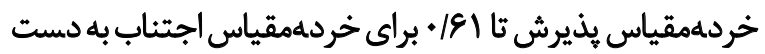

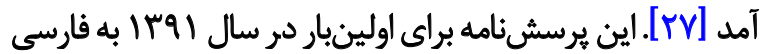

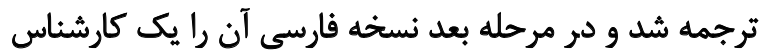

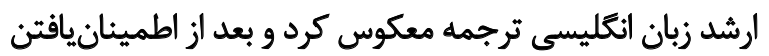

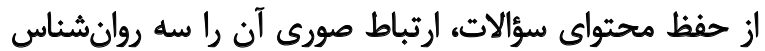

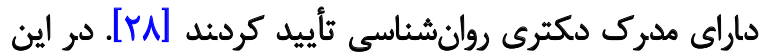

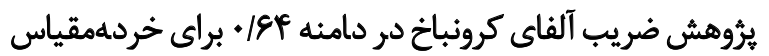

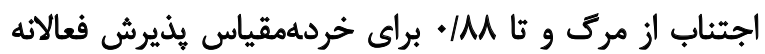

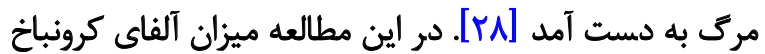

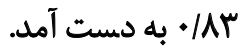

براى طبقلبندى، يردازش و تحليل دادهها و بررسى فرضيه زهاى

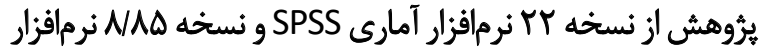
lisrel

3. Weinbergeretal Adjustment Inventory (WAI) 
جدول ا. ويرُّكى هاى جمعيتشئاختى نمونه موردمطالعه

\begin{tabular}{|c|c|c|}
\hline فراوانى (درصد) & & \\
\hline$\Lambda \Delta(\Delta \& / V)$ & نختر & \multirow{3}{*}{ جنسيت } \\
\hline$S \Delta(H T / \Psi)$ & بسر & \\
\hline $10+(1+4)$ & كل كل & \\
\hline if $(q / \pi)$ & 10 & \multirow{6}{*}{ سن } \\
\hline $\operatorname{Mf}(1 \Delta / 9)$ & 18 & \\
\hline$m(r) / q)$ & IV & \\
\hline$r q(r \Delta / A)$ & $M$ & \\
\hline$M F(r T / \Delta)$ & 19 & \\
\hline $\operatorname{lff}(q \Delta / f)$ & كل & \\
\hline$M F(T r / \Delta)$ & بايين & \multirow{4}{*}{ SES } \\
\hline $9 \vee(94 / \pi)$ & هتوسط & \\
\hline $\mathbb{I}(N E)$ & بالا & \\
\hline $\operatorname{lff}(q \Delta / f)$ & كل & \\
\hline$\Delta(r / \mu)$ & ابتلايى & \multirow{4}{*}{ تحصيالات } \\
\hline$P V(r) / 1)$ & راهنمايى & \\
\hline $94(\varepsilon / / 8)$ & دييرستان & \\
\hline Ifos (qध) & كل & \\
\hline$\Delta \mu^{\mu}(r \Delta / 1)$ & I & \multirow{5}{*}{ مرحله سرطان } \\
\hline$r)(r+/ \Delta)$ & II & \\
\hline$r+(1 r / r)$ & III & \\
\hline$M(r) / q)$ & IV & \\
\hline $\operatorname{IrV}(q \cdot / N)$ & كل & \\
\hline
\end{tabular}

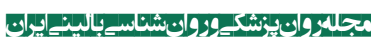

شاخص برازش تطبيقى " در حد مطلوب (بزرختر از • 9/. •) است

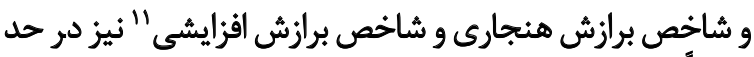

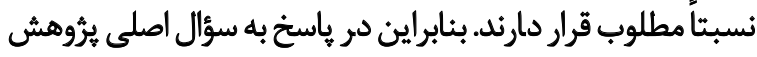

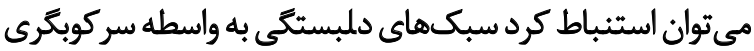

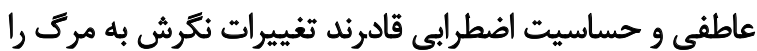
به طور معنى دارى تبيين كنئد.

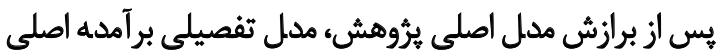

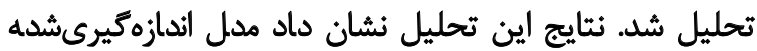

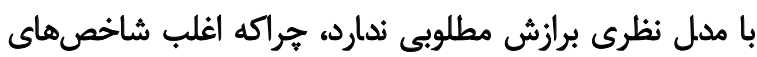

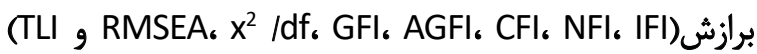
در حد مطلوب قرار نداشتئد. از اين رو براى اصلاح مدل، برخى ،

10. Comparative Fit Index (CFI)

11. Incremental Fit Index (IFI)
مىتوان گفت از جهتى سركوبكرى عاطفى، رابطه بين سبكهاى

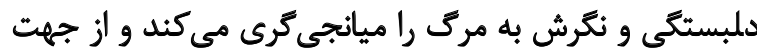

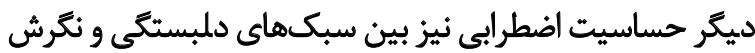
به مرك نقش ميانجى دارد.

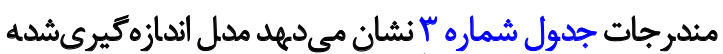

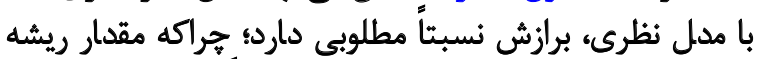

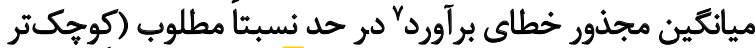

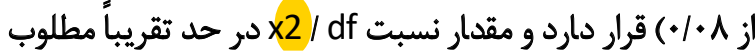

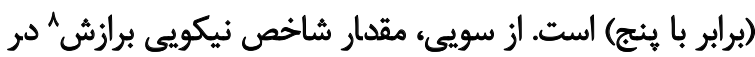

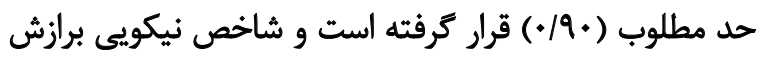

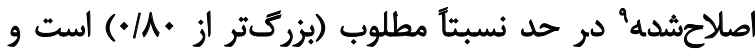

7. Root Mean Square Error of Approximation (RMSEA)

8. Goodness of Fit Index (GFI)

9. Adjusted Goodness of Fit Index (AGFI) 
جدول r. شاخصهاي مركزى و يراكندكيى متغيرها

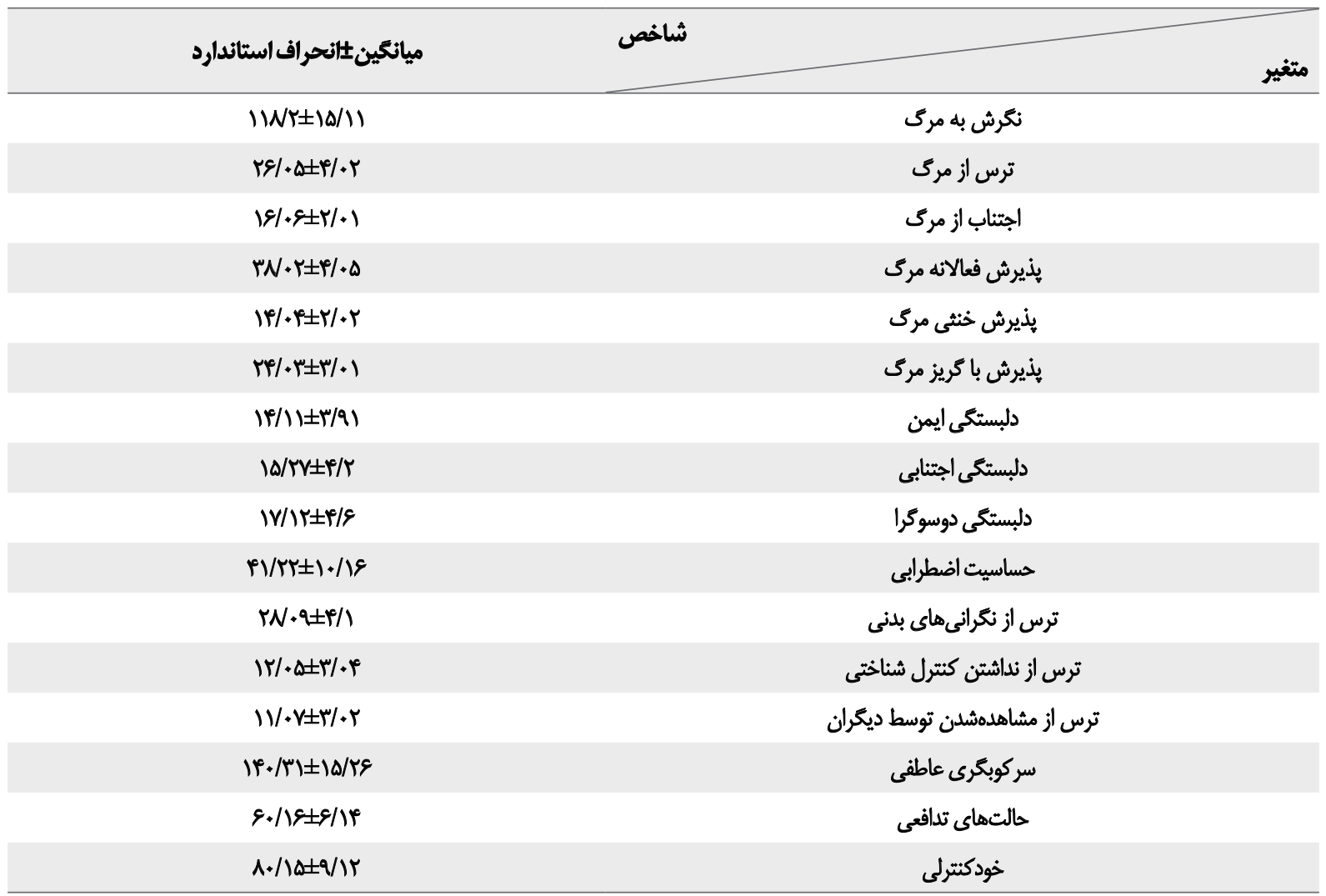

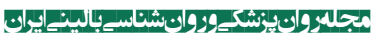

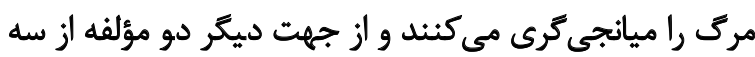

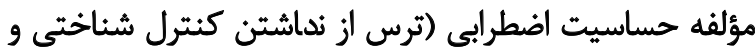

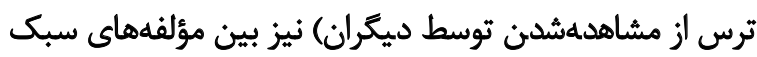

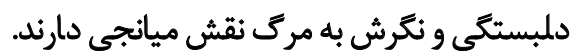

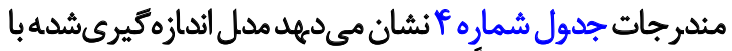

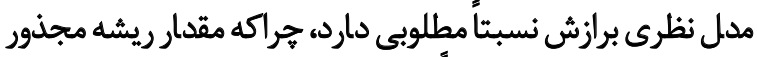

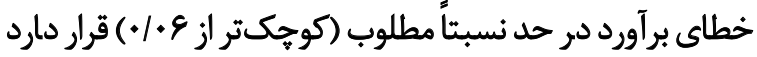

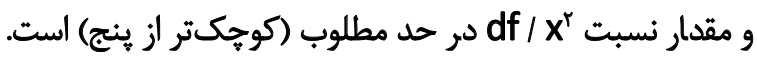

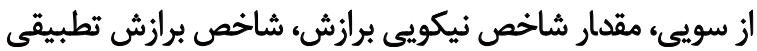

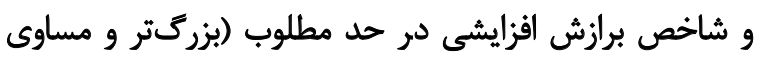

مسيرهاى علّى غيرمعنى دار حذف شدند. مدل اصلاحشده به

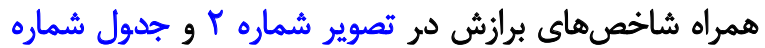

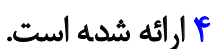
بر اساس يافتههاى مدلي يابى معادلات ساختارى مى توان كفت

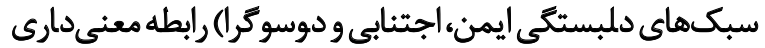

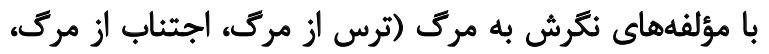

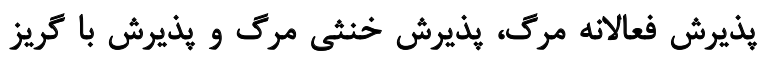

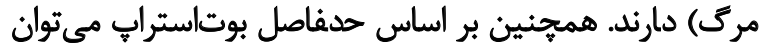

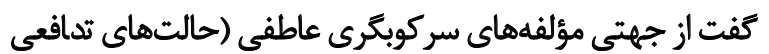

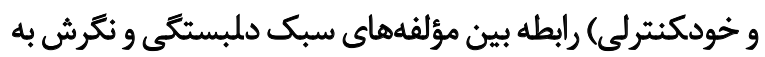

جدول r. شاخصهاي برازندكى مدل ساختارى

\begin{tabular}{|c|c|c|c|c|c|c|c|c|c|}
\hline IFI & NFI & CFI & AGFI & GFI & $\mathbf{P}$ & $\mathbf{X} / / \mathbf{d f}$ & $\mathbf{d f}$ & $\mathbf{X}$ & RMSEA \\
\hline.$/ 19$ &.$/ M$ &.$/ 91$ &.$/ 19$ &.$/ 9$ &.$/ \% 1$ & $\Delta$ & $1 \% \Delta$ & $9 \times \Delta$ &.$/ .9$ \\
\hline
\end{tabular}

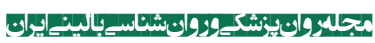

جدول F. شاخصهاى برازندكى مدل ساختارى اصلاحشده

\begin{tabular}{|c|c|c|c|c|c|c|c|c|c|c|}
\hline TLI & IFI & NFI & CFI & AGFI & GFI & $\mathbf{P}$ & $\mathbf{x}^{y} / \mathbf{d f}$ & df & $\mathbf{x}$ & RMSEA \\
\hline$\cdot / A V$ &.$/ q$. & . /AV & . /9Y & $\cdot / M$ &.$/ 91$ & $.1 \ldots 1$ & $f$ & 1.0 & $\mathrm{HT}$. & $\% \Delta$ \\
\hline
\end{tabular}

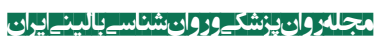




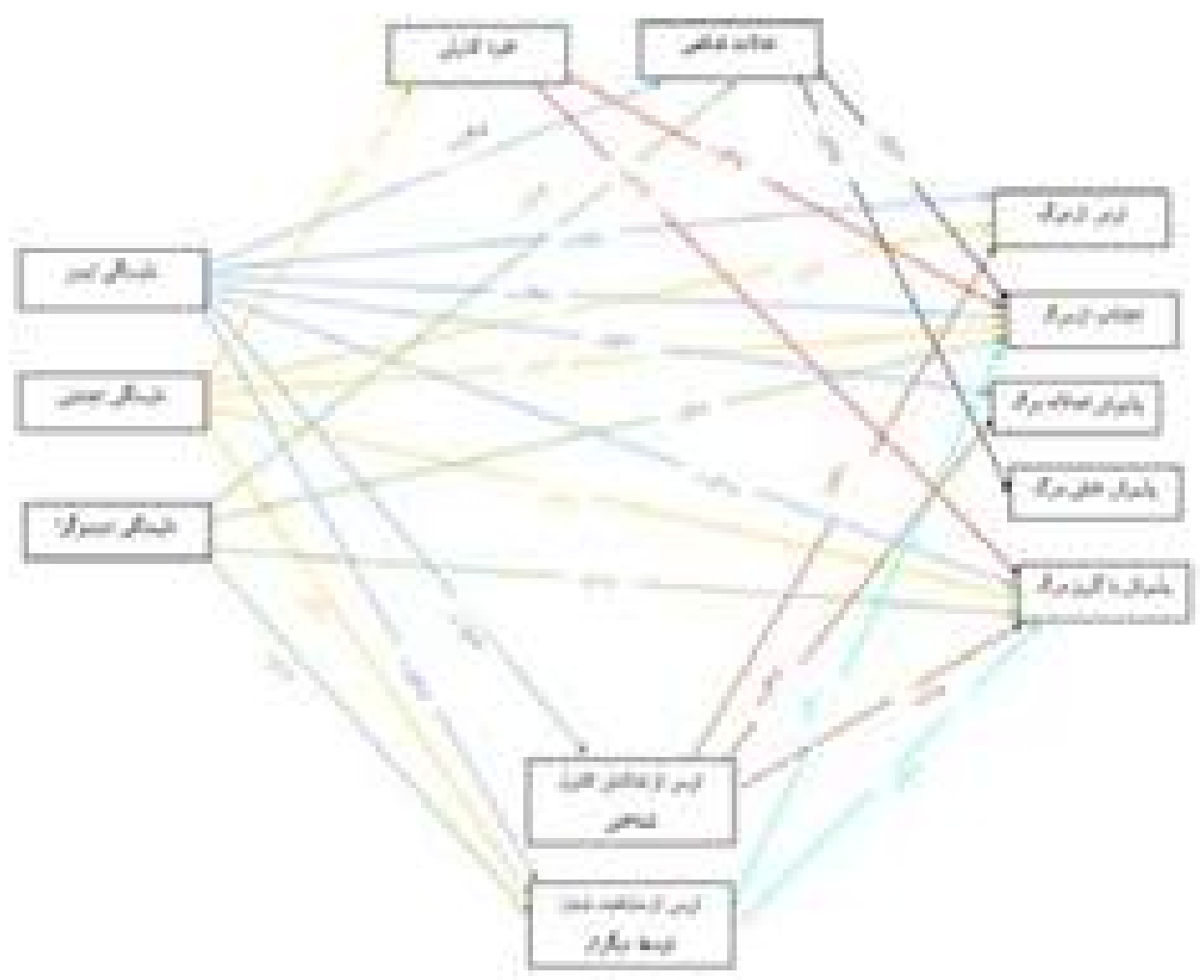

سبكهاي سلكَانه دلبستُى به واسطه مؤلفههاي سركوبى

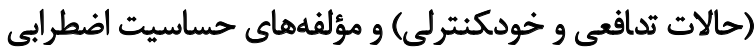

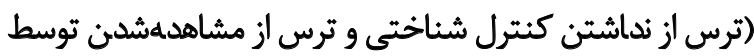

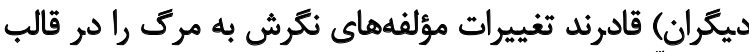

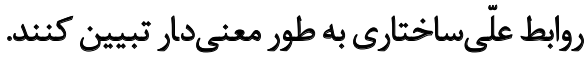

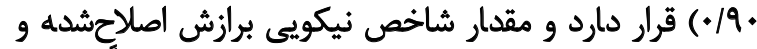

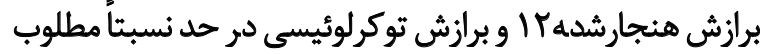

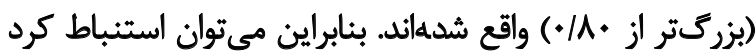

12. Normed Fit Index (NFI)

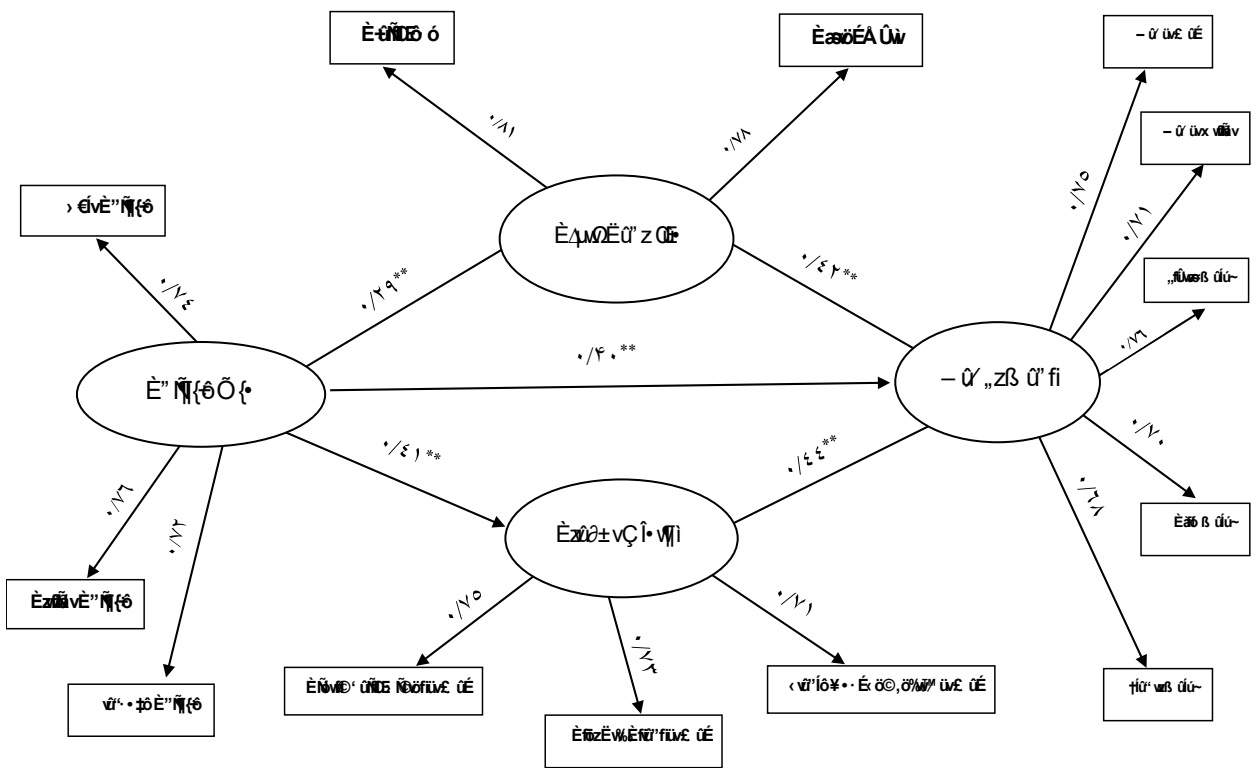


و در فرد احساس امنيت شكل نمي كيرد يا به شكل ضعيف ايجاد

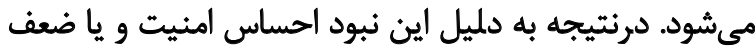

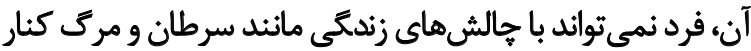

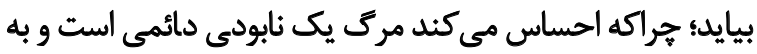

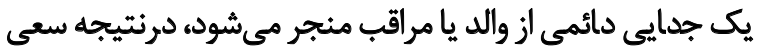

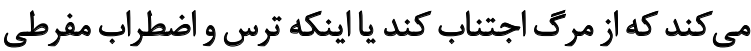

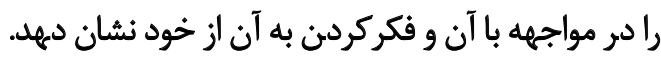

افرادى كه سبك دلمبستى اضطرابى دارند، بر اساس نظر

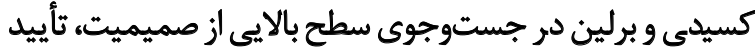

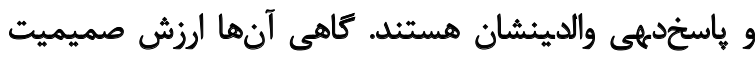

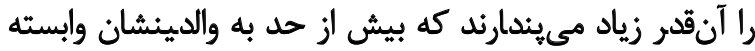

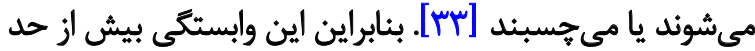

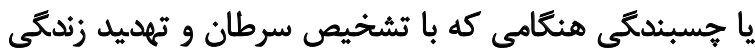

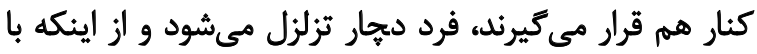

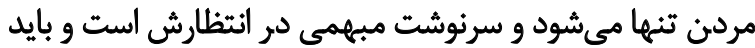

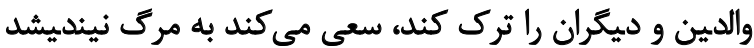

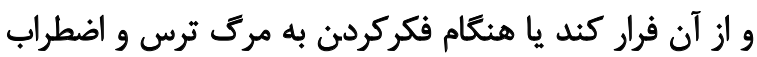

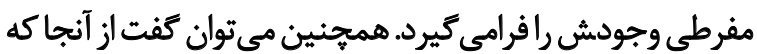

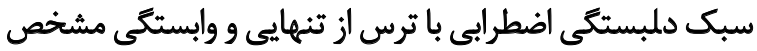

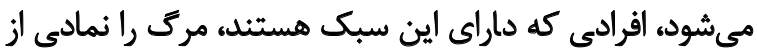

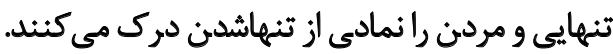

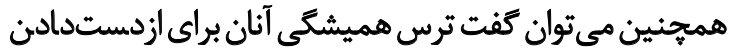

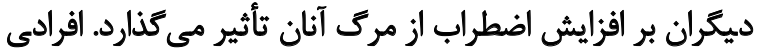

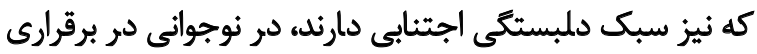

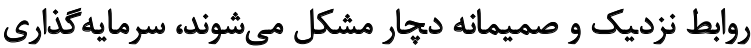

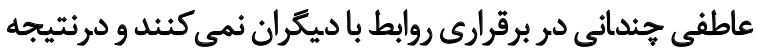

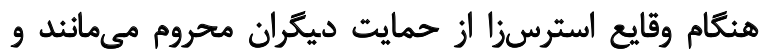

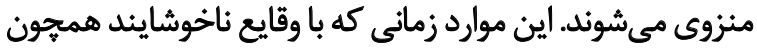

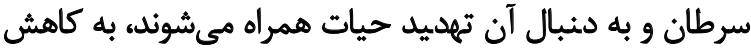

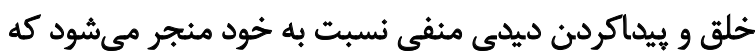

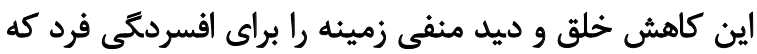

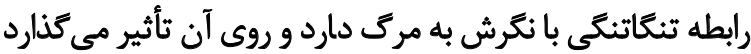

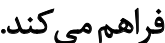

برخى تحقيقات ديكر سطوح كمترى از ترس و اضراب مرى

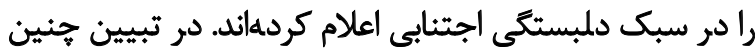

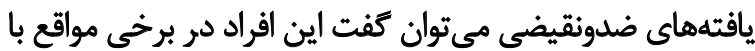

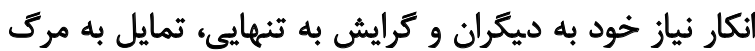

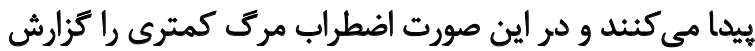

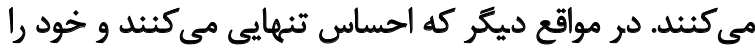
تنها مىيابند، اضطراب مرك بيشترى را كزارش ميكى كنيند. درتبيين اين يافته كه بين دلبستَكى نايمن و وايذيرش باتريز

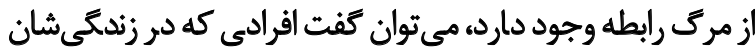

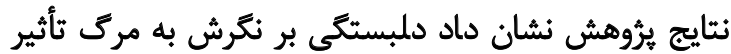

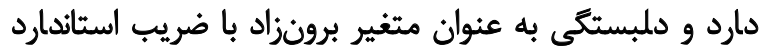

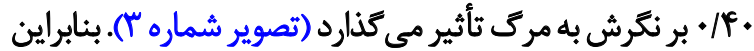

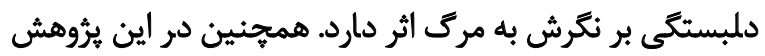

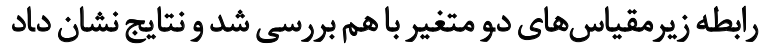

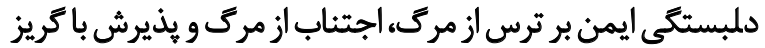

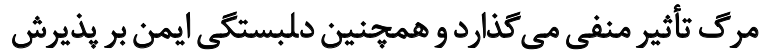

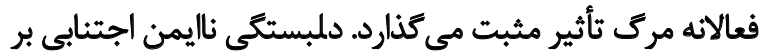

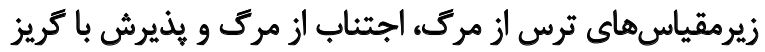

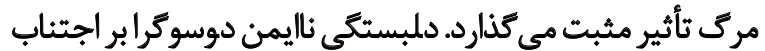

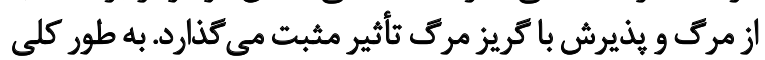

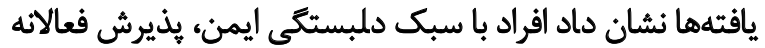

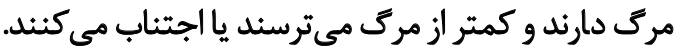

افراد با سبك دلبستكى ناليمن اجتنابى از مرتى مي مترسند و

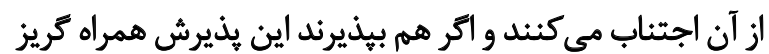

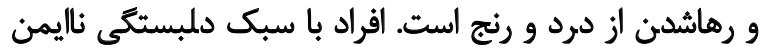

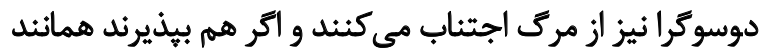

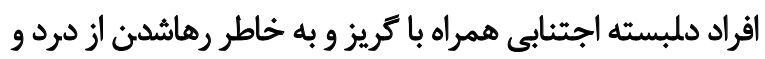

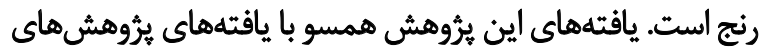

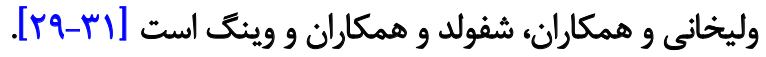

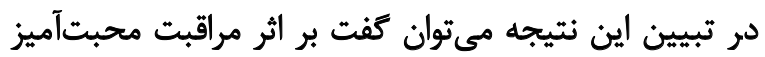

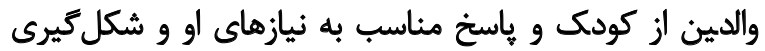

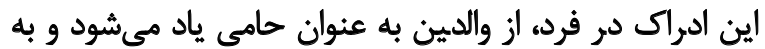

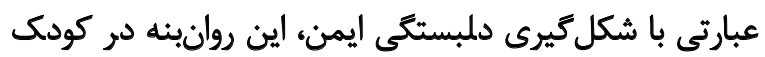

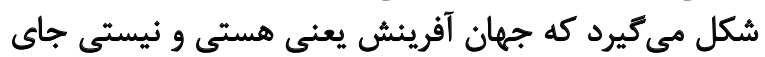
امنى است [بكا.

جنين روانبنهاى زندمَى كودكى را در سالهاى بعدى نوجوانى

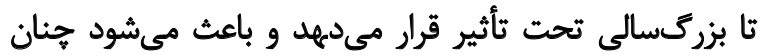

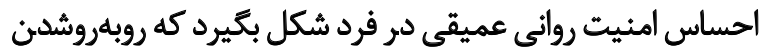

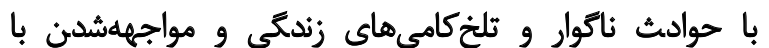

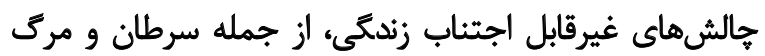

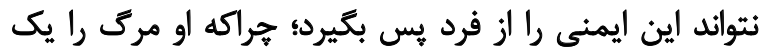

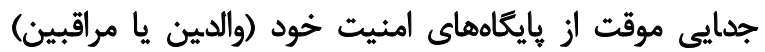

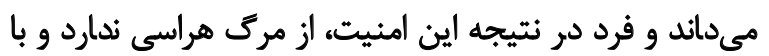

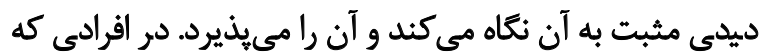

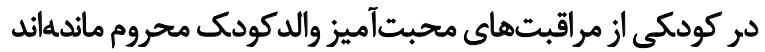

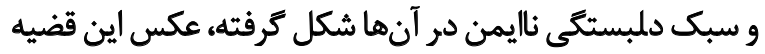

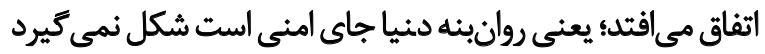

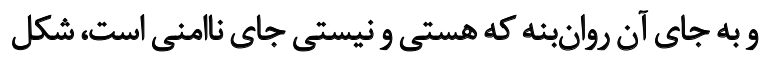

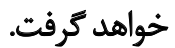

اين روانبنه زندكى بعدى كودك رانيز تحت تأثير قرار مى دهيد 
تنظيم هيجان مثل سركوبكَرى عاطفى، بهداشت روانى فرد را

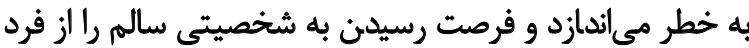

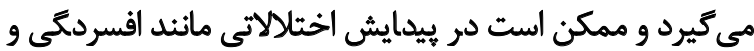

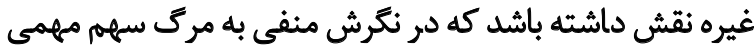

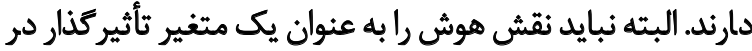

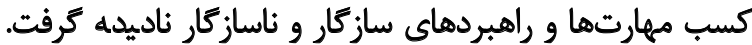

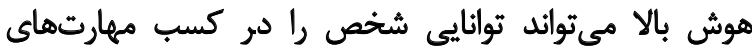

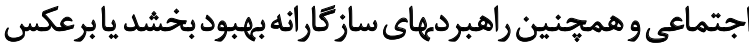

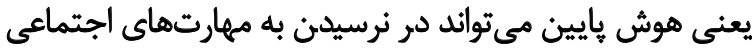

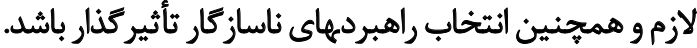

زمانى كه فرد بهويره در سن بايين با تشخيص سرطان روبانه

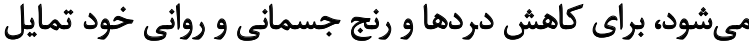

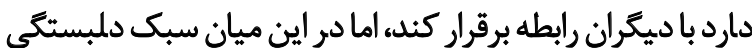

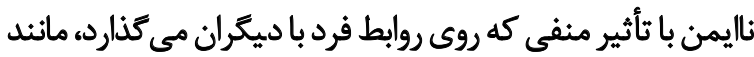

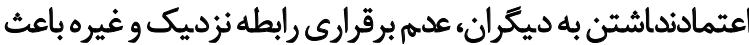

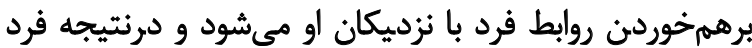

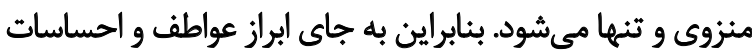

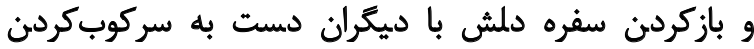

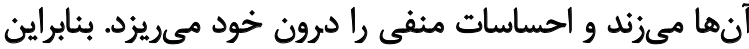

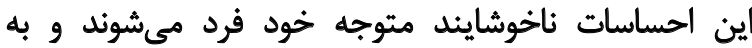

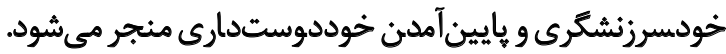

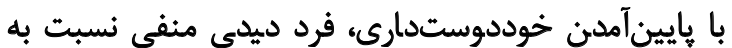

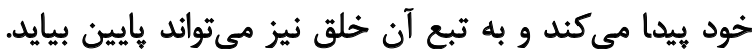

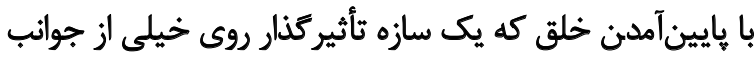

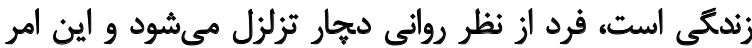

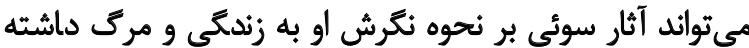

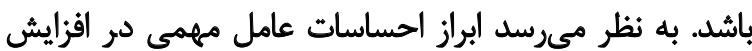

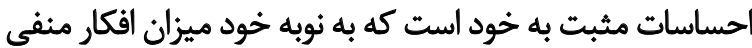

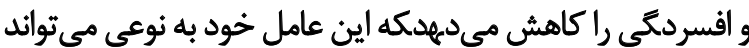

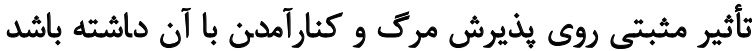

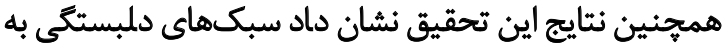

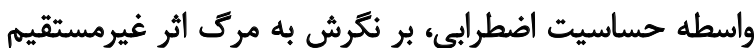

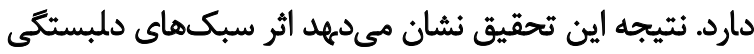

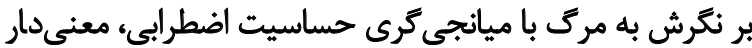

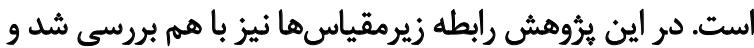

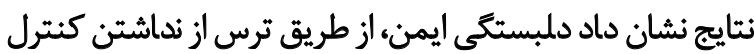

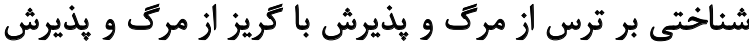

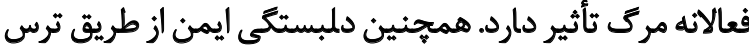

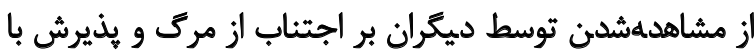

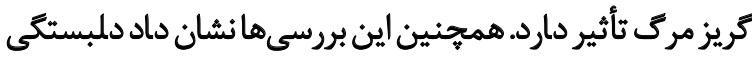

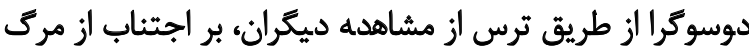

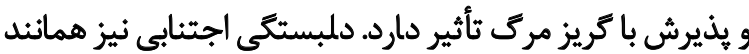

ز زدردها و رنجهاى مزمنى همجهون بيمارى سرطان، رنج مى برند

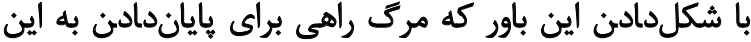

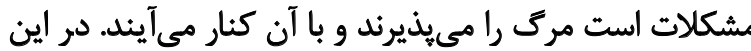

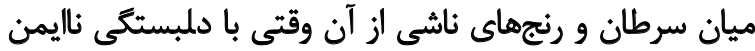
همراه مىشوند به شكل ميرى اين باور منجر مى أشوند.

نتايج اين تحقيق نشان داد سبكهاى دلمبستكى به واسطه

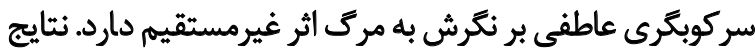

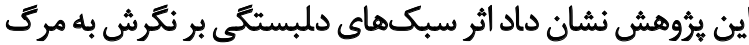
با ميانجى

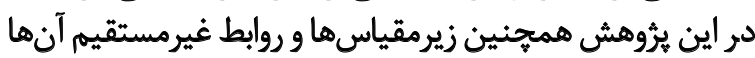

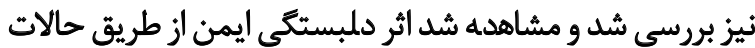

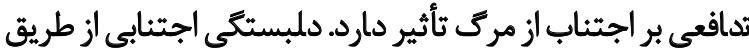

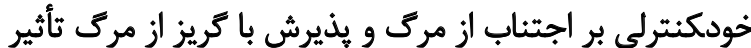

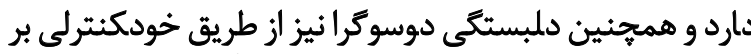

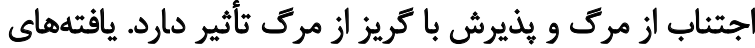

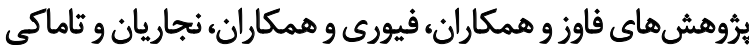

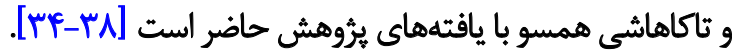

أنجر تبيين اين نتايج مى توان كفت فرد با دلبستكى ناليمن، از

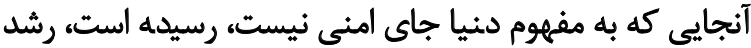

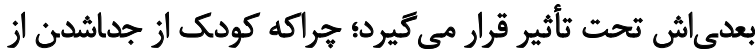

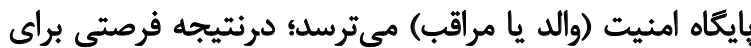

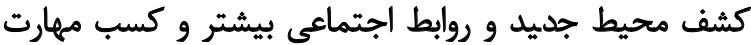

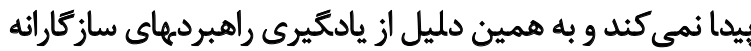

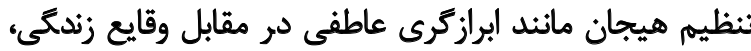

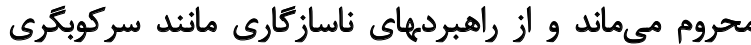

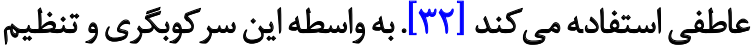

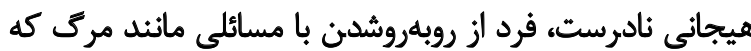

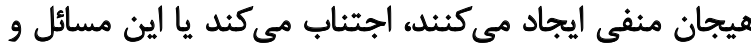

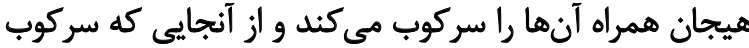

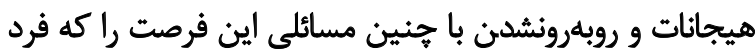

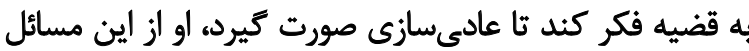

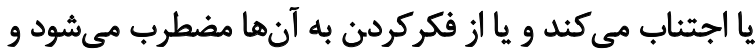

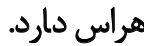

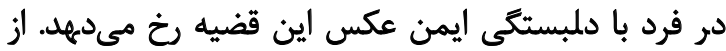

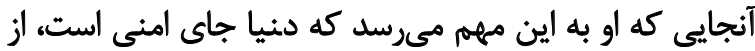

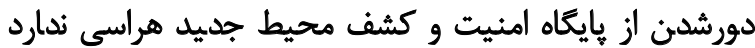

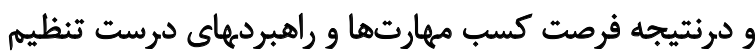

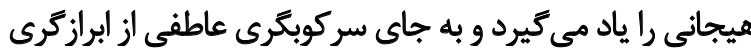

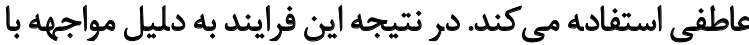

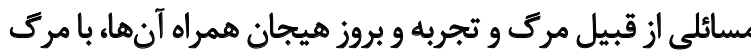

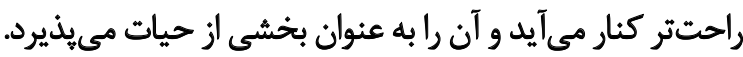
از طرف ديكر مى توان كفت استفاده از راهبردهاى ناسازگارانه 


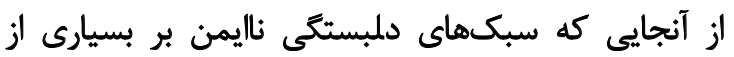

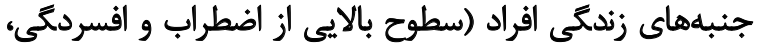

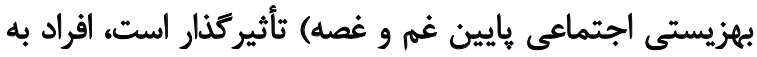

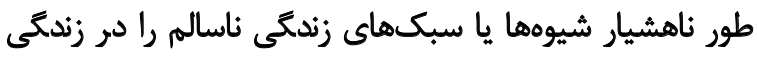

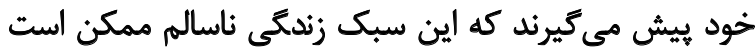

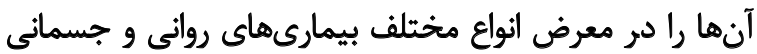

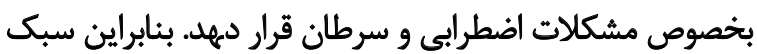

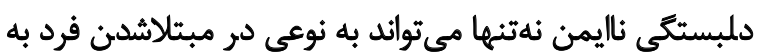

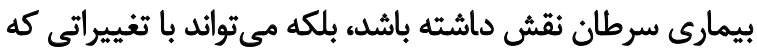

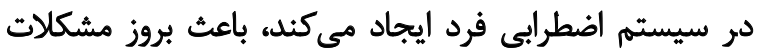

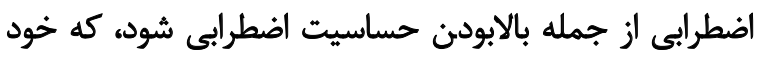

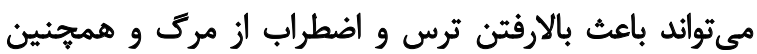

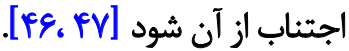

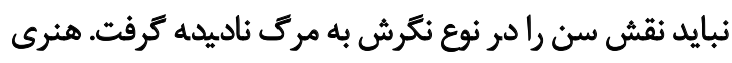

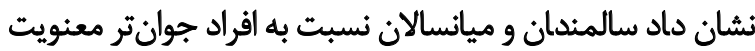

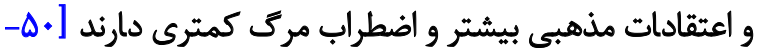

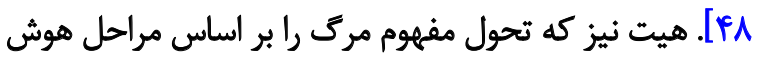

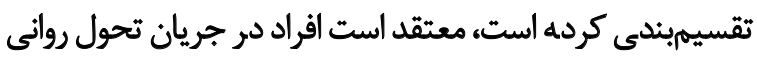

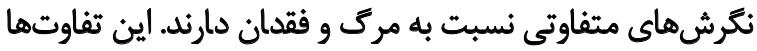

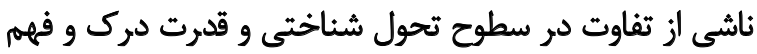

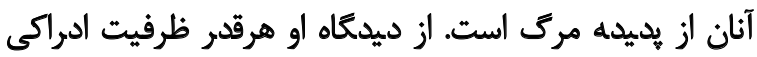

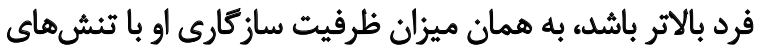

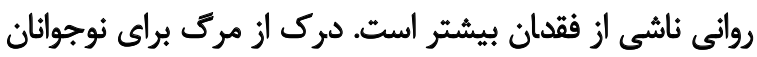

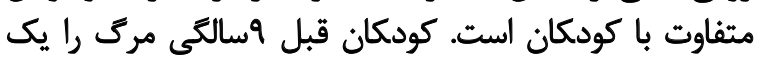

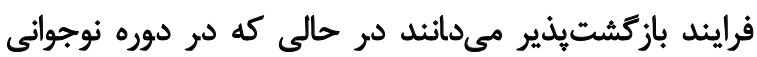

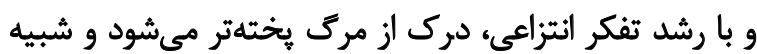

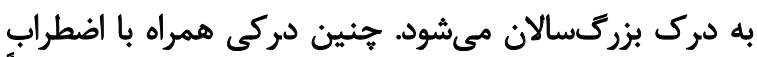

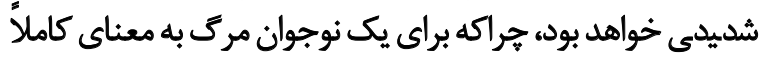

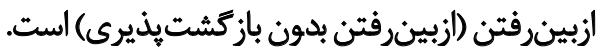

بايد كفت نقش متغيرهايى همجيون مرحله سرطان، نوع

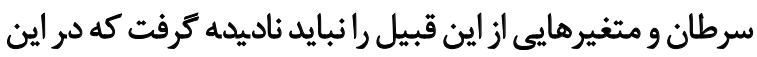

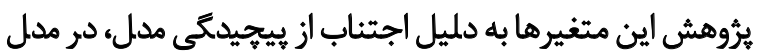

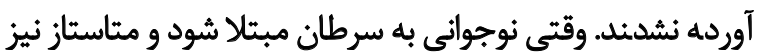

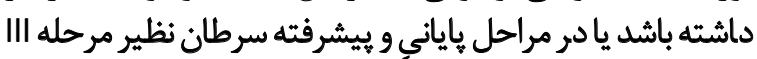

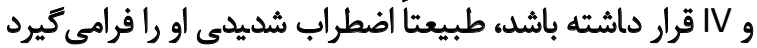

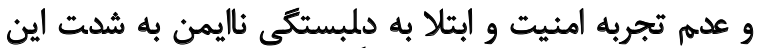

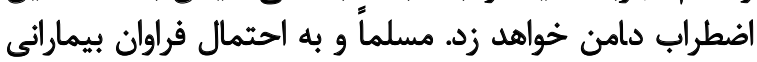

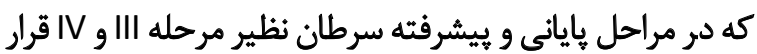

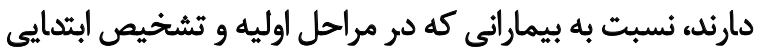

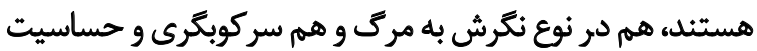

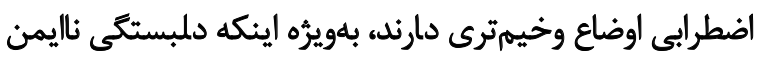
نيز بر اين مشكل (سرطان) دامن بزند. دارند بلدئ.
دلمبستكى دوسوكرا از طريق ترس از مشاهده ديكُران، بر اجتناب از مرك و يذيرش باكريز مرك تأثير دارد.

نتايج يروهش هاي نيلسن و همكاران، سافورد و اسكات و داويلا

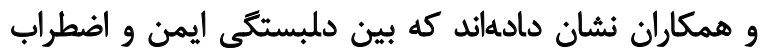

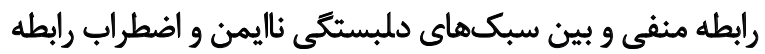

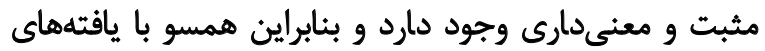

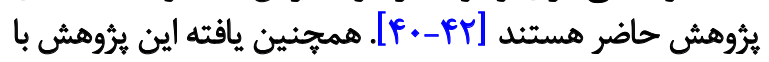

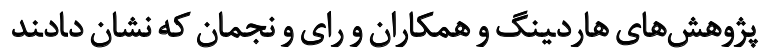

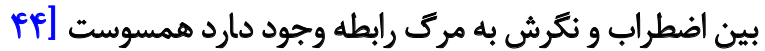

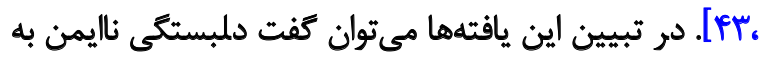

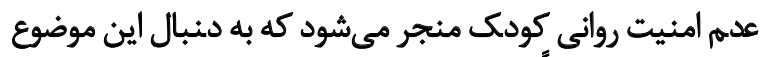

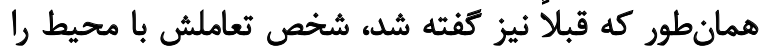

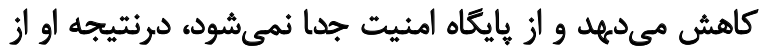

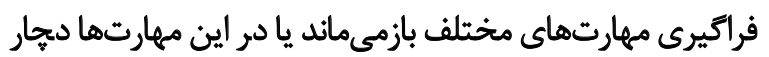
ضعف مي شيود.

ضعف در مهارتها بهتدريج در طول زمان روى رشد كودى

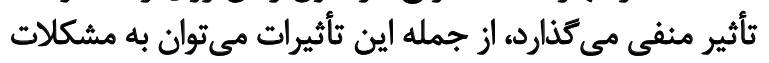

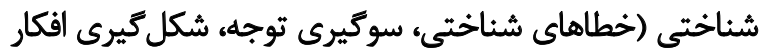

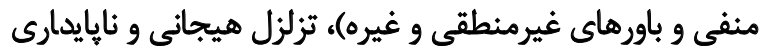

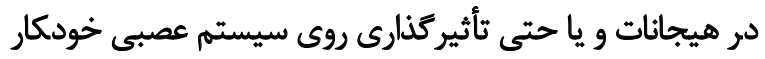

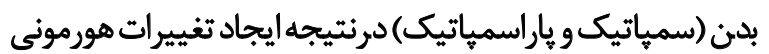

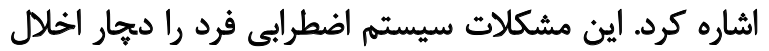
مىكند و فرد به مشكلات اضطرابى از جمله اختلالات اضلات اضطرابى، حساسيت اضطرابى بالاو غيره دجار مي مئرود. در كنار بالابودن حساسيت اضطرابي زماني كه حواديث استرسزايى همجيون سرطان و سرانجام نامعلوم آن آن (درمان يانيان

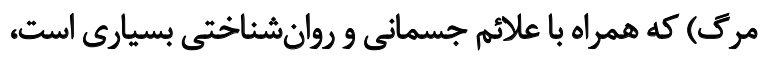

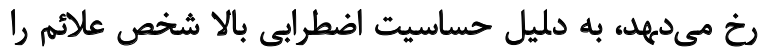

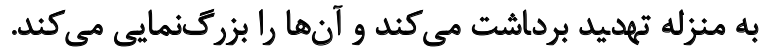

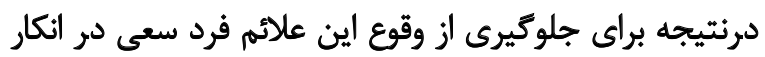

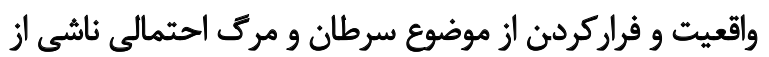

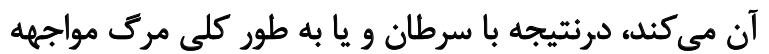

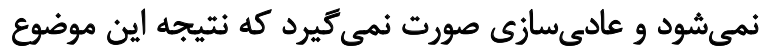

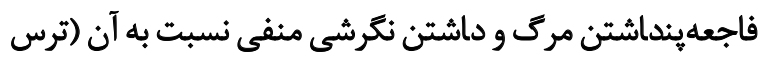

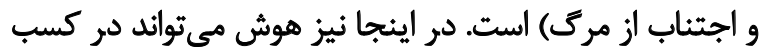

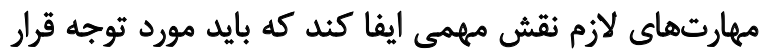
بكيرد. - بهاري مطالعات طولى نشان دادهاند نوع سبك دلبستكى افراد در

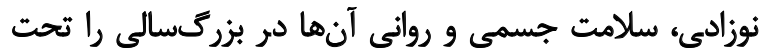

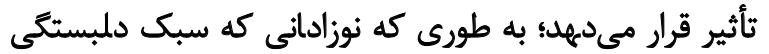

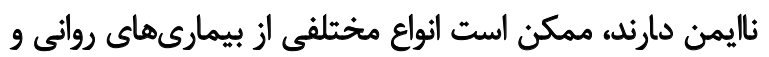

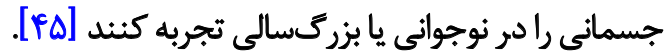


ملاحظات اخلاقى

\section{ييروى أز أصول الخاق يُوهشي}

از همه آزمودنىها براي شركت در يروهش رضايتنامه

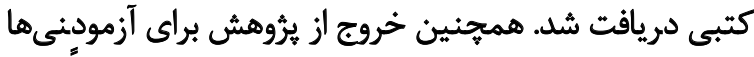

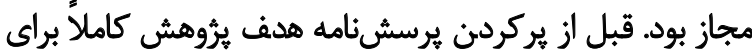

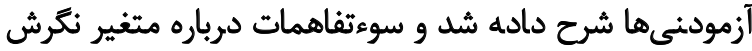

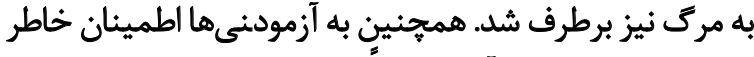

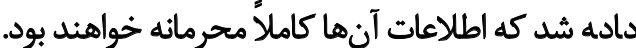

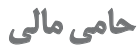

اين يُّوهش هيج كونه حامى مالى ندارد.

\section{مشاركت نويسندكّان}

مفهومسازى: همه نويسندكان؛ روش شناسى: همه نويسندكان؛

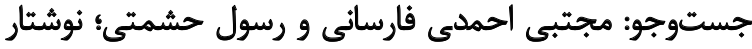

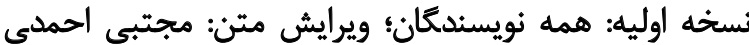

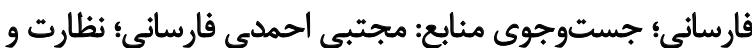

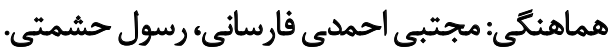
تعارض مثاقع بنابر اظهار نويسندكًان اين مقاله تعارض منافع ندارد.
تئيجليرى

به طور كلى نتايج يُروهش نشان داد سبكهاي دلبستكى به به

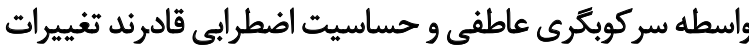

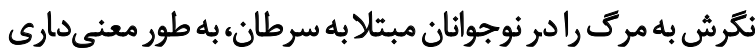

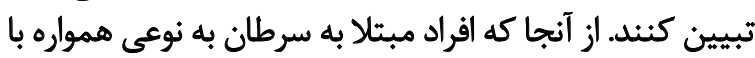

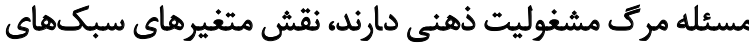

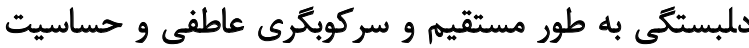

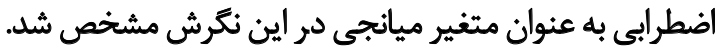

اين يرؤهش داراي محدوديتهايى نيز بود، به دليل اندكسبودن

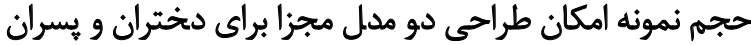

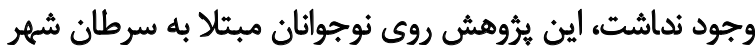

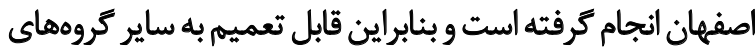

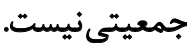

حجم نمونه اندكى، به خاطر كمبودين جامعه بيماران نوجوان

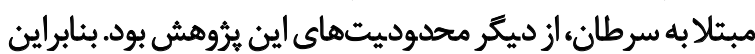

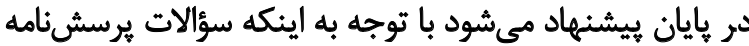

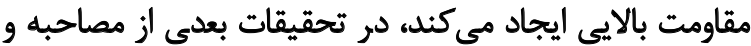

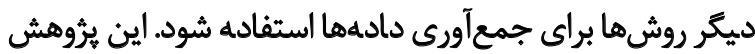

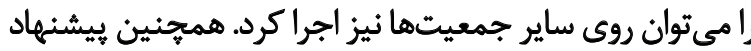

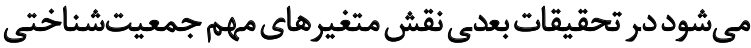

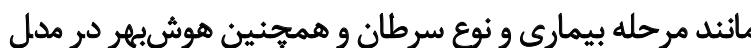

$$
\text { آورده شود. }
$$

همجنين با توجه به نتايج ثروهش بايد علاوه بر سازوكارهايى

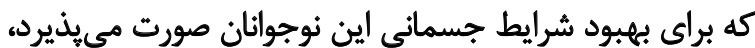

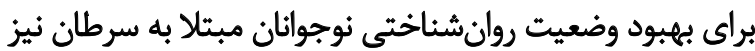

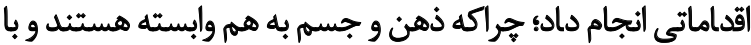

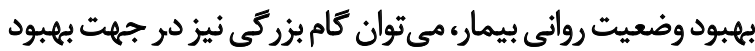

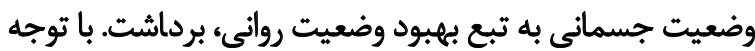

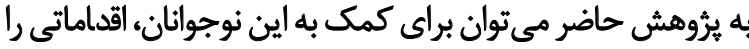

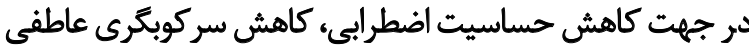

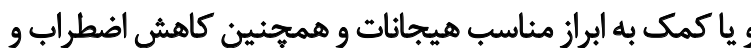
ترس از مرك و همجينين عدم اجتناب از آن انجام دادي

يكى أز رويكردهاي مهمى كه مي تواند هم در كاهش حساسيت

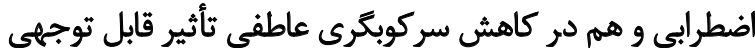

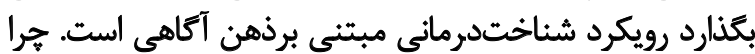

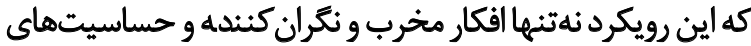

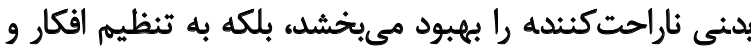

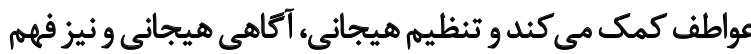

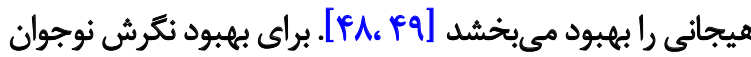

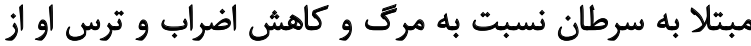

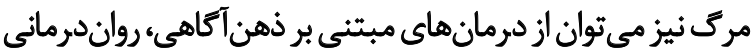

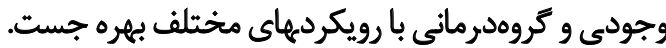




\section{References}

[1] American Cancer Society. Special section: Cancer in children \& adolescents. New York: American Cancer Society; 2014.

[2] Tel H, Tel H, Doğan S. Fatigue, anxiety and depression in cancer patients. Neurology, Psychiatry and Brain Research. 2011; 17(2):42-5. [DOI:10. 1016/j. npbr. 2011. 02. 006]

[3] Robbins RA. Bugen's Coping with Death Scale: Reliability and further validation. Omega-Journal of Death and Dying. 1991; 22(4):287-99. [DOI:10. 2190/HNTD-RWRW-Y3YN-VWX1]

[4] Berg, Laura A. Psychology of growth (from birth to childhood) [Translated by Y Seyed Mohamadi, Persian trans. ]. Tehran: Arasbaran publication; 2007.

[5] Ruiz SK, Harris SJ, Martinez P, Gold PM, Klimes-Dougan B. Young adult's attachment style as a partial mediator between maternal functioning and young adult offsprings' functioning. Journal of Affective Disorders. 2018; 232:393-9. [DOI:10. 1016/j. jad. 2017. 12. 034] [PMID]

[6] Colin VL. McGraw-Hill series in developmental psychology, Human attachment. New York: Mcgraw-Hill Book Company; 1996.

[7] Mund M, Mitte K. The costs of repression: A meta-analysis on the relation between repressive coping and somatic diseases. Health Psychology. 2012; 31(5):640. [DOI:10. 1037/a0026257] [PMID]

[8] Eysenck HJ. Personality, stress and cancer: Prediction and prophylaxis. British Journal of Medical Psychology. 1988; 61(1):57-75. [DOI:10. 1111/j. 2044-8341. 1988. tb02765. x] [PMID]

[9] Mantar A, Yemez B, Alkın T. [Anxiety sensitivity and its place in psychiatric disorders (Turkish)]. Türk Psikiyatri Dergisi. 2011; 22(3):187-93

[10] McNally RJ. Anxiety sensitivity and panic disorder. Biological Psychiatry. 2002; 52(10):938-46. [DOI:10. 1016/S00063223(02)01475-0]

[11] Rapee RM, Medoro L. Fear of physical sensations and trait anxiety as mediators of the response to hyperventilation in nonclinical subjects. Journal of Abnormal Psychology. 1994; 103(4):693-9. [DOI:10. 1037//0021-843X. 103. 4. 693] [PMID]

[12] Marshall GN, Miles JN, Stewart SH. Anxiety sensitivity and PTSD symptom severity are reciprocally related: evidence from a longitudinal study of physical trauma survivors. Journal of $\mathrm{Ab}$ normal Psychology. 2010; 119(1):143-50. [DOI:10. 1037/a0018009] [PMID] [PMCID]

[13] Valikhani A, Firouzabadi A. [Examining death anxiety within the framework of attachment styles in cancer patients: A comparative study (Persian)]. Health Psychology. 2016; 5(18): 119-130.

[14] Farsi Z, Dehghan Nayeri N, Negarandeh R. [The role of personal factors in coping of adults with acute leukemia undergoing hematopoietic stem cell transplantation (Persian)]. Nurse and Physician Within War. 2015; 3(8):58-69.

[15] Esmaeili R, Ahmadi F, Mohammadi E, Tirgari Seraj A. [Life threatening: The most important concern of patients confronting cancer diagnosis (Persian)]. Hayat. 2013; 18(5):12-22.

[16] Mitchell AJ, Ferguson DW, Gill J, Paul J, Symonds P. Depression and anxiety in long-term cancer survivors compared with spouses and healthy controls: A systematic review and meta- analysis. The Lancet Oncology. 2013; 14(8):721-32. [DOI:10. 1016/ S1470-2045(13)70244-4]

[17] Fuller-Thomson E, Baird SL, Dhrodia R, Brennenstuhl S. The association between Adverse Childhood Experiences (ACEs) and suicide attempts in a population-based study. Child: Care, Health and Development. 2016 Sep;42(5):725-34 [DOI:10. 1111/ cch. 12351] [PMID]

[18] Garssen B. Psychological factors and cancer development: Evidence after 30 years of research. Clinical Psychology Review. 2004; 24(3):315-38. [DOI:10. 1016/j. cpr. 2004. 01. 002] [PMID]

[19] Kaplow JB, Gipson PY, Horwitz AG, Burch BN, King CA Emotional suppression mediates the relation between adverse life events and adolescent suicide: Implications for prevention Prevention Science. 2014; 15(2):177-85. [DOI:10. 1007/s11121-013 0367-9] [PMID] [PMCID]

[20] Bolton P, Bass J, Betancourt T, Speelman L, Onyango G, Clougherty KF, et al. Interventions for depression symptoms among adolescent survivors of war and displacement in northern Uganda: A randomized controlled trial. Jama. 2007; 298(5):519-27. [DOI:10.1001/jama. 298. 5. 519] [PMID]

[21] Sarafino EP, Smith TW. Health psychology: Biopsychosocial interactions. Hoboken: John Wiley \& Sons; 2014

[22] Ghanbari B, Hatami A, Esmaieli A, Farahbakhsh K. [On the relationship among parenting styles, attachment styles and marital commitment in arried female students of Allameh Tabatabai University (Persian)]. Women and Society. 2011; 2(3):39-60.

[23] Floyd M, Garfield A, LaSota MT. Anxiety sensitivity and worry. Personality and Individual Differences. 2005; 38(5):1223-9. [DOI:10. 1016/j. paid. 2004. 08. 005]

[24] Reiss S, Peterson RA, Gursky DM, McNally RJ. Anxiety sensitivity, anxiety frequency and the prediction of fearfulness. Behaviour Research and Therapy. 1986; 24(1):1-8. [DOI:10. 1016/00057967(86)90143-9]

[25] Mashhadi A, Gasempoor A, Akbari E, ElBeigi R, Hasanzadeh $\mathrm{SH}$. [The role of anxiety sensitivity and emotion regulation in prediction of social anxiety disorder in students (Persian)]. Knowledge \& Research in Applied Psychology. 2013; 14(2): 90-100.

[26] Saeedi Z, Ghorbani N, Sarafraz MR, Shoar TK. A bias of selfreports among repressors: Examining the evidence for the validity of self-relevant and health-relevant personal reports. International Journal of Psychology. 2018; 15(59):335-47. [DOI:10. 1002/ ijop. 12560

[27] Wong PT, Reker GT, Gesser G. Death attitude profile-revised: A multidimensional measure of attitudes toward death. In Neimeyer RA, editors. Death anxiety Handbook: Research, Instrumentation, and Application. Abingdon: Taylor \& Francis; 1994

[28] Basharpoor S, Vojoodi B, Atarod N. [Relationship between religious orientation and attitude toward death with quality of life and signs of physicalization in women (Persian)]. Health Psychology. 2011;3(10):80-97.

[29] Valikhani A, Sarafraz MR, Moghimi P. Examining the role of attachment styles and self-control in suicide ideation and death anxiety for patients receiving chemotherapy in Iran. Psycho-Oncology. 2018; 27(3):1057-60. [DOI:10. 1002/pon. 4466] [PMID]

[30] Scheffold K, Philipp R, Koranyi S, Engelmann D, SchulzKindermann F, Härter M, et al. Insecure attachment predicts 
depression and death anxiety in advanced cancer patients. Palliative \& Supportive Care. 2018; 16(3):308-16. [DOI:10. 1017/ S1478951517000281] [PMID]

[31] Wing MK. Predicting death anxiety with gratitude and friendship attachment: A correlational study [BSc. thesis]. Hong Kong: Hong Kong Baptist University; 2011.

[32] Bowlby J. Elite psychology and education-attachment theory [K Khoshabi, E Aboohamzeh Persian trans. ] Tehran: Danjeh. 2007.

[33] Cassidy J, Berlin LJ. The insecure/ambivalent pattern of attachment: Theory and research. Child Development. 1994; 65(4):97191. [DOI:10. 1111/j. 1467-8624. 1994. tb00796. x] [PMID]

[34] Favez N, Cairo Notari S, Antonini T, Charvoz L. Attachment and couple satisfaction as predictors of expressed emotion in women facing breast cancer and their partners in the immediate post-surgery period. British Journal of Health Psychology. 2017; 22(1):169-85. [DOI:10. 1111/bjhp. 12223] [PMID]

[35] Fiori KL, Buthmann J, Denckla CA. Crying and attachment style: The role of romantic relationships. Journal of Social, Behavioral, and Health Sciences. 2017; 11(1):133-46. [DOI:10. 5590/ JSBHS. 2017. 11.1.09]

[36] Najarian Z, Arfaei F, Tamannaiifar M. [Prediction of coping styles \& assertiveness based on attachment stylesamong male vs. female in Kashan University Students (Persiaqn)]. New Thoughts on Education. 2015; 11(3):87-109.

[37] Tamaki K, Takahashi J. The relationship between adult attachment style and social skills in terms of the four-category model of attachment style. International Journal of Humanities and Social Science. 2013; 3(19):84-90.

[38] Dattel AR, Neimeyer RA. Sex differences in death anxiety: Testing the emotional expressiveness hypothesis. Death Studies. 1990; 14(1):1-1. [DOI:10. 1080/07481189008252341]

[39] Kehtary L, Heshmati R, Pour Sharifi H. [Investigating structural pattern of depression based on experiential avoidanceand emotional repression: The mediating role of self-compassion (Persian)]. Iranian Journal of Psychiatry and Clinical Psychology. 2018; 24(3):284-297.

[40] Nielsen SK, Lønfeldt N, Wolitzky-Taylor KB, Hageman I, Vangkilde S, Daniel SI. Adult attachment style and anxiety- the mediating role of emotion regulation. Journal of Affective Disorders. 2017; 218:253-9. [DOI:10. 1016/j. jad. 2017. 04. 047] [PMID]

[41] Safford, Scott Matthew. "The relationship of attachment style and cognitive style to depression, anxiety and negative affectivity. 2004; 2(2):573-80.

[42] Davila J, Ramsay ME, Stroud CB, Steinberg SJ. Attachment as vulnerability to the development of psychopathology. In: Hankin Bl, Abela JRZ, editors. Development of psychopathology: A vulnerability-stress perspective. Thousand Oaks: Sage Publications; 2005. [DOI:10. 4135/9781452231655. n9]

[43] Harding SR, Flannelly KJ, Weaver AJ, Costa KG. The influence of religion on death anxiety and death acceptance. Mental Health, Religion \& Culture. 2005; 8(4):253-61. [DOI:10. 1080/13674670412331304311]

[44] Ray JJ, Najman J. Death anxiety and death acceptance: A preliminary approach. OMEGA-Journal of Death and Dying. 1975; 5(4):311-5. [DOI:10. 2190/MHEL-88YD-UHKF-E98C]
[45] Puig J, Englund MM, Simpson JA, Collins WA. Predicting adult physical illness from infant attachment: A prospective longitudinal study. Health Psychology. 2013; 32(4):409. [DOI:10. 1037/ a0028889] [PMID] [PMCID]

[46] Porter LS, Keefe FJ, Davis D, Rumble M, Scipio C, Garst J. Attachment styles in patients with lung cancer and their spouses: associations with patient and spouse adjustment. Supportive Care in Cancer. 2012; 20(10):2459-66. [DOI:10. 1007/s00520-011-1367-6] [PMCID]

[47] Domingue P. An examination of attachment styles and distress among parents who have lost a child to cancer [PhD dissertation] Washington: The Catholic University of America; 2011

[48] Paterniti A. A pilot study comparing the efficacy of a mindfulness-based program to a skills-training program in the treatment of test anxiety. Ann Arbor: ProQuest; 2007.

[49] Goldin PR, Gross JJ. Effects of Mindfulness-Based Stress Reduction (MBSR) on emotion regulation in social anxiety disorder. Emotion. 2010; 10(1):83-91. [DOI:10.1037/a0018441] [PMID] [PMCID]

[50] Henrie JA. Religiousness, future time perspective, and death anxiety among adults. Morgantown: West Virginia University; 2010. 
This Page Intentionally Left Blank 